\title{
INTERDEPENDENCE OF ELECTRIC DISCHARGE AND MAGNETOROTATIONAL INSTABILITY IN PROTOPLANETARY DISKS
}

\section{$\operatorname{AUTHOR}(S)$ :}

Muranushi, Takayuki; Okuzumi, Satoshi; Inutsuka, Shu-ichiro

\section{CITATION:}

Muranushi, Takayuki ...[et al]. INTERDEPENDENCE OF ELECTRIC DISCHARGE AND MAGNETOROTATIONAL INSTABILITY IN PROTOPLANETARY DISKS. The Astrophysical Journal 2012, 760(1): 56.

\section{ISSUE DATE:}

2012-11

URL:

http://hdl.handle.net/2433/163491

\section{RIGHT:}

(c) 2012. The American Astronomical Society.; この論文は出版社版であ りません。引用の際には出版社版をご確認ご利用ください。; This is not the published version. Please cite only the published version. 


\title{
Interdependence of Electric Discharge and The Magnetorotational Instability in Protoplanetary Disks
}

\author{
Takayuki Muranushi \\ The Hakubi Center for Advanced Research, Kyoto University, Sakyo-ku, Kyoto, 606-8502, Japan; \\ muranushi.takayuki.3r@kyoto-u.ac.jp \\ Satoshi Okuzumi \\ Nagoya University, Furo-cho, Chikusa-ku, Nagoya, 464-8601, Japan; okuzumi@nagoya-u.ac.jp \\ Shu-ichiro Inutsuka \\ Nagoya University, Furo-cho, Chikusa-ku, Nagoya, 464-8601, Japan; inutsuka@nagoya-u.ac.jp
}

\begin{abstract}
We study how the magnetorotational instability (MRI) in protoplanetary disks is affected by the electric discharge caused by the electric field in the resistive MHD. We have performed three-dimensional shearing box simulations with various values of plasma beta and electrical breakdown models. We find the self-sustainment of the MRI in spite of the high resistivity. The instability gives rise to the large electric field that causes the electrical breakdown, and the breakdown maintains the high ionization degree required for the instability. The condition for this self-sustained MRI is set by the balance between the energy supply from the shearing motion and the energy consumed by the Ohmic dissipation. We apply the condition to various disk models and study where the active, self-sustained, and dead zones of MRI are. In the fiducial minimum-mass solar nebula (MMSN) model, the newly-found sustained zone occupies only the limited volume of the disk. In the late-phase gas-depleted disk models, however, the sustained zone occupies larger volume of the disk.
\end{abstract}

Subject headings: Dust — planets and satellites:formation — planetary systems: protoplanetary disks - MHD - instabilities

\section{Introduction}

Protoplanetary disks are the sites of planet formation. The disk turbulence greatly affects the mutual sticking of the planetesimals, their settlement to the disk midplane. The turbulence is the source of the angular momentum transfer in the disk that causes gas accretion and migration of the planetesimal onto the central star. Thus understanding the evolution of the turbulence within protoplanetary disks is an essential step both in the studies of the disk evolution and the planet formation.
The magnetorotational instability (MRI) is considered to be the major source of turbulence in many types of accretion disks including protoplanetary disks (Balbus \& Hawley (1998) and references therein). One of the distinct properties of the protoplanetary disks compared to other accretion disks is that the major parts of the protoplanetary disks are only weakly ionized, and the magnetic diffusivity affects the MRI (Sano et al. (1998); Fleming et al. (2000).) The low ionization degree is due to their low temperature and high number density of the dust component. 
In protoplanetary disks, MRI and the dust components affect each other. The turbulence is one of the source for the relative velocities of the colliding dust (Ormel \& Cuzzi 2007; Brauer et al. 2008), and contributes both on dust growth and disruption (Blum \& Wurm 2008; Wada et al. 2008; Güttler et al. 2010; Wettlaufer 2010). On the other hand, dust particles in protoplanetary disks are the major sites of charged particle recombination, and thereby influences the ionization degree of the disk. (Sodha et al. 2009; Umebayashi \& Nakano 2009; Grach et al. 2010)

The dead zone can occupy a large volume of a protoplanetary disk, especially in the presence of abundant small dust grains (Gammie 1996; Sano et al. 2000; Ilgner \& Nelson 2006). However, various electric discharge mechanisms in protoplanetary disks have been proposed (Horanyi et al. 1995; Desch \& Cuzzi 2000; Muranushi 2010) which may provide higher ionization degree compared to the values predicted by the dust-absorption equilibrium models, resulting in the increased MRI activity in the disk. They consider the electron avalanche process, an exponential growth in the number of conducting electrons that takes place when the kinetic energy of the electrons exceeds the ionization energy of a neutral gas molecule. The result is electrostatic breakdown, the lowering of the resistivity of the fluid and electric discharge, increase of the electric current through the fluid.

Moreover, a model is proposed where the MRI itself provides sufficient ionization (Inutsuka \& Sano 2005, hereafter IS05). IS05 have shown that that the electric field typically generated by the protoplanetary disk turbulence is strong enough to drive the electrons away from the thermal Maxwell-Boltzmann distribution. Those energetic electrons contained in electric current cause the electric discharge and maintains the ionization degree high enough for the MRI to survive. IS05 have also shown that the energy supply from the shearing motion is about 30 times larger than the energy required to maintain the sufficient number of electrons in the presence of standard dust grains.

However, IS05 have studied only one-zone models, and with only one set of parameters typical to 1 AU of the disk. In this work, we extend the model IS05 to a local, 3D simulations of protoplanetary disks and study the interaction of the
MRI with the discharge ionization. We also apply the model to global models of protoplanetary disks and study where and when in the disk the self-sustainment of MRI takes place.

This paper is organized as follows. in $\S 2$, we perform the numerical simulations of the MRI in unstratified, three-dimensional shearing-boxes along the lines of Hawley et al. (1995, hereafter HGB95) with the nonlinear Ohmic diffusivity added. In $\S 3$, We analyze the activity of the MRI in the protoplanetary disk, using the method of Sano et al. (2000, hereafter SMUN00). To calculate the ionization degree in the disk we use the method proposed by Okuzumi (2009, hereafter O09). $\$ 4$ is devoted to conclusions and discussions. Table 1 lists the symbols frequently used in this paper.

\section{Simulations of The MRI with nonlinear Ohm's law}

\subsection{Numerical Setup}

There are three diffusion terms in MHD; they are Ohmic diffusion, Hall diffusion and ambipolar diffusion. In protoplanetary disks any one of the three modes can be the dominant mode depending on dust and gas density (e.g. Wardle 2007), and interaction between the different modes may alter the MRI (Wardle \& Salmeron 2012). In this paper we only focus on the Ohmic diffusion because it is the most studied one in the context of the MRI. We leave the treatment of other diffusion modes for future studies.

The electric discharge taken into account, we construct a simple model of the discharge as follows, in terms of an appropriate $\eta_{0}$ and $J_{\text {crit }}$ :

$$
\begin{aligned}
\mathbf{E}^{\prime} & =\frac{4 \pi}{c^{2}} \eta(J) \mathbf{J}, \\
\eta(J) & =\eta_{0} \quad \text { if } \quad J<J_{\text {crit }}, \\
& =\frac{J_{\text {crit }}}{J} \eta_{0} \quad \text { if } \quad J>J_{\text {crit }} .
\end{aligned}
$$

This nonlinear diffusivity model states that the electric field on the fluid co-moving frame never exceeds a critical value, $E_{\text {crit }}^{\prime}=4 \pi c^{-2} \eta_{0} J_{\text {crit }}$, thus the magnetic diffusivity $\eta$ varies depending on electric current $J$, and Ohm's law become nonlinear.

Note that the smallest space scale dealt in this paper is of order of $10^{-2}$ AU. The actual scale 


\begin{tabular}{lclc}
\hline \hline Symbol & Value (Dimension) & \multicolumn{1}{c}{ Definition } & Location \\
\hline$\rho$ & $\left(\mathrm{g} \mathrm{cm}^{-3}\right)$ & Gas density & $(4)$ \\
$\mathbf{v}$ & $\left(\mathrm{cm} \mathrm{s}^{-1}\right)$ & Gas velocity & $(5)$ \\
$\mathbf{B}$ & $\left(\mathrm{g}^{1 / 2} \mathrm{~cm}^{-1 / 2} \mathrm{~s}^{-1}\right)$ & Magnetic field & $(6)$ \\
$P$ & $c_{s}^{2} \rho$ & Pressure with isothermal equation of state & $(7)$ \\
$\mathbf{E}$ & $\left(\mathrm{g}^{1 / 2} \mathrm{~cm}^{-1 / 2} \mathrm{~s}^{-1}\right)$ & Electric field in the lab frame & $(8)$ \\
$\mathbf{E}^{\prime}$ & $\left(\mathrm{g}^{1 / 2} \mathrm{~cm}^{-1 / 2} \mathrm{~s}^{-1}\right)$ & Electric field in the comoving frame & $(? ?)$ \\
$\mathbf{J}$ & $\left(\mathrm{g}^{1 / 2} \mathrm{~cm}^{-3 / 2} \mathrm{~s}^{-1}\right)$ & Electric current & $(10)$ \\
$\eta_{0}$ & $\left(\mathrm{~cm}^{2} \mathrm{~s}^{-1}\right)$ & Linear coefficient and - & \\
$J_{\text {crit }}$ & $\left(\mathrm{g}^{1 / 2} \mathrm{~cm}^{-3 / 2} \mathrm{~s}^{-1}\right)$ & - critical current for extended Ohm's law & $(2)$ \\
$P_{0}$ & $\left(\mathrm{~g} \mathrm{~cm}^{-1} \mathrm{~s}^{-2}\right)$ & Initial pressure & \\
$\beta$ & $2 c_{s}^{2} / v_{\mathrm{Az}}{ }^{2}$ & Plasma beta & \\
$B_{z 0}$ & $\sqrt{\beta} B_{\text {eqp }}$ & Initial, vertical net magnetic field & $(16)$ \\
$H$ & $c_{s} / \Omega$ & Disk scale-height & $(15)$ \\
$B_{\text {eqp }}$ & $\sqrt{8 \pi P_{0}}$ & The nondimensionalization unit of magnetic field & $(13)$ \\
$J_{\text {eqp }}$ & $c B_{\text {eqp }} / 4 \pi H$ & The nondimensionalization unit of current & $(14)$ \\
$R_{\mathrm{M}}$ & $v_{\mathrm{Az}} / \eta_{0} \Omega$ & Magnetic Reynolds number & $\S 2.2$ \\
& & & \\
$f_{\Sigma}$ & 1.0 & Surface Density Multiplier & $(39)$ \\
$q$ & $3 / 2$ & Power Law Index of the Surface Density & $(39)$ \\
$a_{d}$ & $0.1 \mu \mathrm{m}$ & Radius of Solid Dust Particle & $(46)$ \\
$f_{d}$ & 0.01 & Dust to Gas Ratio & $(49)$ \\
\hline
\end{tabular}

Table 1: The list of symbols used in this paper.

of the discharge structures can be much smaller than this. The estimate for the lower limit of the size of such structures is their thickness, which is of the order of 5000 times electron mean free path (Pilipp et al. 1992).

However, we can derive the macroscopic discharge model from the microscopic discharge relation $\left|E^{\prime}\right|<E_{\text {crit }}^{\prime}$ that holds everywhere in the plasma. Since e.g. the $x$-component of the discretized electric field $\left\langle E^{\prime}{ }_{x}\right\rangle$ is obtained from the line integral of the real field over the discretization length $\Delta V$,

$$
\begin{aligned}
\left|\left\langle E^{\prime}{ }_{x}\right\rangle\right| & =\left|\frac{\int E^{\prime}{ }_{x} d V}{\Delta V}\right| \\
& \leq \frac{\int\left|E^{\prime}{ }_{x}\right| d V}{\Delta V} \\
& \leq \frac{\int E^{\prime}{ }_{\text {rit }} d V}{\Delta V} \\
& =E^{\prime}{ }_{\text {crit }} .
\end{aligned}
$$

Therefore, we can use Eqs. (1), (2) as a "coarsegrained model" where we can interpret $E^{\prime}, J$ as spatial averages. If electrical breakdown occurs in a scale smaller than grid size, the spatially averaged electric field is smaller than $E_{\text {crit }}^{\prime}$. Thus, in general, the electrical breakdown may occur even in the region where $\left\langle E^{\prime}{ }_{\text {crit }}\right\rangle$ is smaller than $E_{\text {crit }}^{\prime}$. Therefore, if we adopt equations (1) and (2), we may underestimate, but not overestimate, the occurrence of electric discharges.

We adopted a local, Kepler-rotation shearing box that has radial (x), azimuthal (y) and vertical $(\mathrm{z})$ axes, and solved the following resistive magnetohydrodynamic (MHD) equations numerically:

$$
\begin{aligned}
\frac{\partial \rho}{\partial t}+\nabla \cdot(\rho \mathbf{v})= & 0, \\
\frac{\partial \mathbf{v}}{\partial t}+\mathbf{v} \cdot \nabla \mathbf{v}= & -\frac{1}{\rho} \nabla\left(P+\frac{B^{2}}{8 \pi}\right)+\frac{1}{4 \pi \rho}(\mathbf{B} \cdot \nabla \mathbf{B}) \\
& -2 \boldsymbol{\Omega} \times \mathbf{v}+3 \Omega^{2} x \hat{\mathbf{x}}, \\
\frac{\partial \mathbf{B}}{\partial t}= & -c \nabla \times \mathbf{E},
\end{aligned}
$$

with isothermal EOS

$$
P=c_{s}^{2} \rho, .
$$


Had we used the adiabatic EOS, the internal energy would have kept growing as the linear function of time (HGB95). Therefore, we use the isothermal EOS to approximate the steady state attained by the cooling processes present in the protoplanetary disks.

The nonlinear Ohm's law reads:

$$
\begin{aligned}
\mathbf{E} & =-\frac{1}{c} \mathbf{v} \times \mathbf{B}+\frac{4 \pi}{c^{2}} \eta(J) \mathbf{J}, \\
\mathbf{J} & =\frac{c}{4 \pi} \nabla \times \mathbf{B},
\end{aligned}
$$

Here, we have studied three different models for nonlinear diffusivity $\eta(J)$. In addition to our fiducial model(fid), Eqs. (1) (2), we have studied the following two models:

$$
\begin{aligned}
& (\mathrm{p} 2): \eta(J)=\left(1+\left(\frac{J_{\text {crit }}}{J}\right)^{-2}\right)^{-\frac{1}{2}}, \\
& (\mathrm{p} 4): \eta(J)=\left(1+\left(\frac{J_{\text {crit }}}{J}\right)^{-4}\right)^{-\frac{1}{4}} .
\end{aligned}
$$

The electric field as functions of current density in these three models are shown in Figure 1.

Following HGB95, we set up our numerical initial conditions as follows. We use the disk scaleheight $H$ as the unit length. The box size is $\left(L_{x}, L_{y}, L_{z}\right)=(1,2 \pi, 1)$. First, we set the average values $\rho_{0}=1$ and $P_{0}=10^{-6}$ to every mesh, and let fluid velocity to be at rest in shearing box frame; $\left(v_{x}, v_{y}, v_{z}=0,-(3 / 2) \Omega x, 0\right)$. Here, $c_{s}=10^{-3}$, and also $\Omega=10^{-3}$.

Next, we introduce random perturbations in density, pressure, and velocity. The density and pressure perturbations are in proportion so that the isothermal EOS is met, and the amplitude is $\delta \rho / \rho_{0}=\delta P / P_{0}=2.5 \times 10^{-2}$. We perturb the velocity component-wise, with the amplitude $\delta v_{i}=5 \times 10^{-3} c_{s}$ for each.

We use the following units of magnetic field and electric current:

$$
\begin{aligned}
& B_{\text {eqp }} \equiv \sqrt{8 \pi P_{0}}, \\
& J_{\text {eqp }} \equiv \frac{c B_{\text {eqp }}}{4 \pi H},
\end{aligned}
$$

and the scale height

$$
H=\frac{c_{s}}{\Omega} .
$$

We set uniform magnetic field in the $z$-direction, and express the initial field strength by the plasma beta,

$$
\beta \equiv B_{\text {eqp }}{ }^{2} / B_{z 0}{ }^{2}=8 \pi P_{0} / B_{z 0}{ }^{2} .
$$

The plasma beta satisfy the following relation between the sound speed $c_{s}$ and the Alfvén velocity along the magnetic field $v_{\mathrm{Az}}$ :

$$
\beta=\frac{2 c_{s}^{2}}{v_{\mathrm{Az}}^{2}} .
$$

We define the magnetic Reynolds number as:

$$
R_{\mathrm{M}} \equiv v_{\mathrm{Az}}^{2} / \eta_{0} \Omega
$$

using the Alfvén velocity $v_{\mathrm{Az}}=B_{z 0} / \sqrt{4 \pi \rho}$ set by the initial vertical magnetic field. This is in accordance with SMUN00 and IS05 while some literature adopts different definition (e.g. $R_{\mathrm{M}} \equiv$ $c_{s}^{2} / \eta_{0} \Omega$ in Fleming et al. 2000) .

We have used Athena (Gardiner \& Stone 2005, 2008), an open-source MHD code for our simulations.

\subsection{Simulations Procedure}

We vary the initial magnetic field strength and the diffusivity models, and we classify each set of parameters as either Oactive zone, $\times$ dead zone, or $\triangle$ sustained zone. The experiment method and the definition of the three classes are given in this section.

The parameters we have investigated are the initial vertical field strength (represented by plasma $\beta$ ), the linear diffusivity (represented by magnetic Reynolds number $R_{\mathrm{M}}$ ), and the critical current $J_{\text {crit. }}$. The range of the survey was $400 \leq \beta \leq 25600,0.002 \leq R_{\mathrm{M}} \leq 2$ and $0.01 \leq J_{\text {crit }} / J_{\text {eqp }} \leq 100$. In addition, the limiting cases of $R_{\mathrm{M}}=\infty$ and $J_{\text {crit }} / J_{\text {eqp }}=\infty$, that respectively correspond to ideal MHD models and linear Ohm's law models, are studied for comparison with the literature.

For each value of $\beta$, we prepared the initial condition as described in section 2.1, and continued the simulation for 10 orbits $(t=20 \pi / \Omega)$, at first with magnetic diffusivity turned off $(\eta(J)=0)$. While running the simulations, we created restart data for every periodic points $(t=2 n \pi / \Omega$ where $n$ 
is an integer). The condition allowed the MRI to grow and saturate in about 5 orbits $(t=10 \pi / \Omega)$.

Then, for each pair of $\left(R_{\mathrm{M}}, J_{\text {crit }} / J_{\text {eqp }}\right)$, we turned on the diffusivity and re-started the simulation either from the initial laminar flow $(t=$ 0 ) or the saturated MRI states at 8,9 and 10 orbit $(t=16 \pi / \Omega, 18 \pi / \Omega, 20 \pi / \Omega)$. We numerically evolved them until they reach 20 orbit $(t=$ $40 \pi / \Omega)$. The reason why we have adopted three different MRI saturated initial conditions $(t=$ $16 \pi / \Omega, 18 \pi / \Omega, 20 \pi / \Omega)$ for each set of the parameters is that a 'turbulent initial condition' is not unique; therefore we need to test if our results depend on the choice of the initial condition or not.

During each simulation run, we recorded the space averages of physical quantities as the functions of time, such as magnetic energy density $B^{2}$, the Reynolds and Maxwell stress $\rho v_{x} \delta v_{y}$, $-B_{x} B_{y} / 4 \pi$, and the squared current $J^{2}$. After the simulations we studied the time average of the quantities. For a physical quantity $A$, we denote its space and time average by $\langle A\rangle$ and $\bar{A}$, respectively. Their definitions are as follows:

$$
\begin{aligned}
\langle A\rangle & \equiv \frac{\int d x \int d y \int d z A}{\int d x \int d y \int d z}, \\
\bar{A} & \equiv \frac{\int d t A}{\int d t} .
\end{aligned}
$$

The space average is taken for the entire computational domain $\left(0<x<L_{x}, 0<y<L_{y}, 0<\right.$ $\left.z<L_{z}\right)$, numerical resolution is $\left(N_{x}, N_{y}, N_{z}\right)=$ $(64,128,64)$, and the time average is taken for the last five orbit $(30 \pi<t<40 \pi)$ unless otherwise mentioned. The statistics for the important sets of parameters are presented at the end of the paper.

Using the average values, we classify each set of the parameter $\left(\beta, R_{\mathrm{M}}, J_{\text {crit }} / J_{\text {eqp }}\right)$ as follows (c.f. Table 2). First, a parameter is in Oactive zone if the MRI is observed both in the simulation started from laminar flow as well as in all of

\begin{tabular}{c|cc} 
zone & from laminar? & from ideal MRI? \\
\hline Oactive & unstable & unstable \\
$\times$ dead & stable & stable \\
$\triangle$ sustained & stable & unstable
\end{tabular}

Table 2: The zone names and the meaning of the symbols in Figures 3 and 4 . the three simulations started from the saturated MRI states. Second, a parameter is in $\times$ dead zone if the instability is not observed neither in the simulation started from laminar flow as well as in any of the three simulations started from the saturated MRI states. Finally, a parameter is in $\triangle$ sustained zone, if the MRI is observed at in all the three simulations started from the saturated MRI states, but not at the end of the simulation started from laminar flow.

\subsection{The Result of Shearing-Box Simula- tions}

The typical behavior of the current for the active zone, dead zone and sustained zone are in Figure 2. From the simulations we have observed that the three classes (active, dead and sustained) are exhaustive: that the runs started from 8,9 and 10 orbit always agree in terms of the classification; and that if the MRI dies when starting from saturated initial condition, it also does not activate starting from laminar initial condition.

To classify the active, dead and sustained zone, we need to assess the magnetorotational instability of the system, so we introduce the following criteria for quantitative assessment. We say that the system is magnetorotationally unstable if the averaged current $\left\langle J^{2}\right\rangle^{1 / 2}$ is greater than $0.1 J_{\text {eqp }}$, and stable if otherwise. Here, the time average is taken for the last five orbit $(30 \pi<t<40 \pi)$. We have learned from the simulations that the quantity $\left\langle J^{2}\right\rangle^{1 / 2}$ is a good indicator for the stability, since it either fluctuates around mean value $\left\langle J^{2}\right\rangle^{1 / 2} \simeq 10 J_{\text {eqp }}$ (unstable) or go under $\left\langle J^{2}\right\rangle^{1 / 2}<$ $0.1 J_{\text {eqp }}$ almost monotonically with little vibration (stable), and there is no ambiguity between the two; c.f. Figure 2.

However, as we make $R_{\mathrm{M}}$ smaller, the diffusion timescale becomes shorter, and the wall clock time for the simulations until $t=40 \pi$ becomes impractically larger. Therefore, for the parameter range $R_{\mathrm{M}}<0.01$, we terminate the simulations at $1.5 \times 10^{6}$ cycles and determine the class by extrapolations.

Figures 3 and 4 show the result of our parameter survey. We found that sustained zone exist - the MRI does exhibit hysteresis behavior for a certain set of parameters.

To study possible influences of the numeri- 
restart from $t=0$

(a)

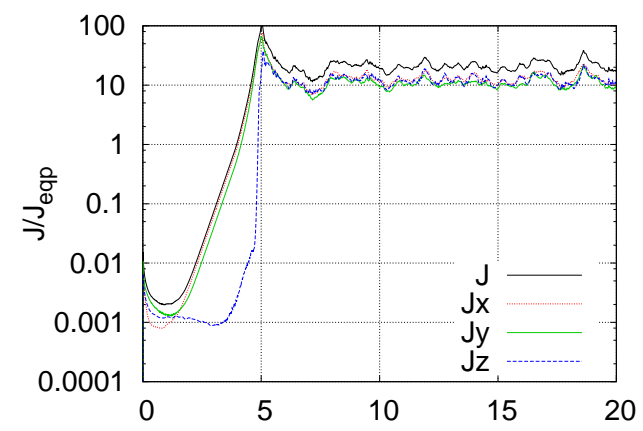

(b)

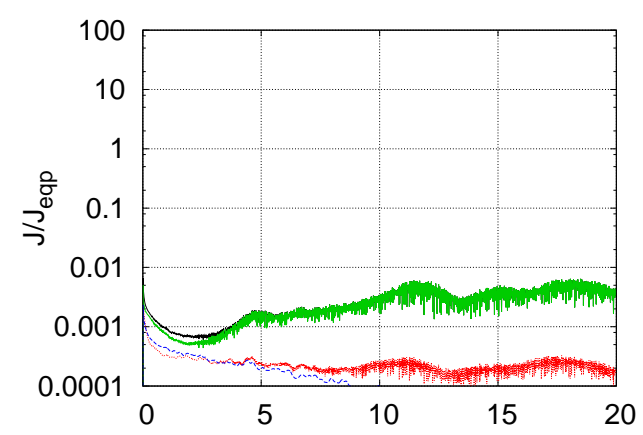

(c)

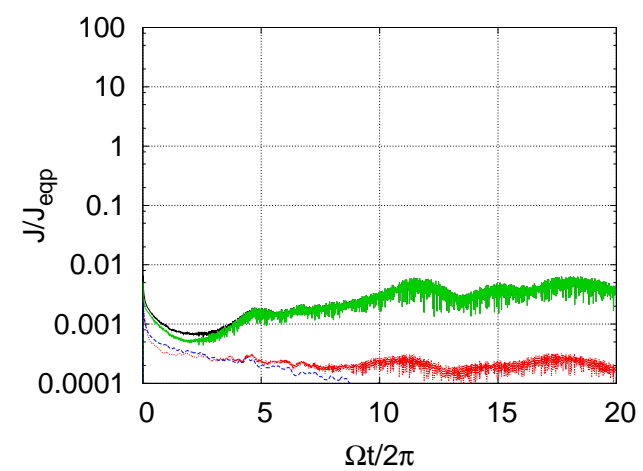

restart from $t=16 \pi / \Omega$
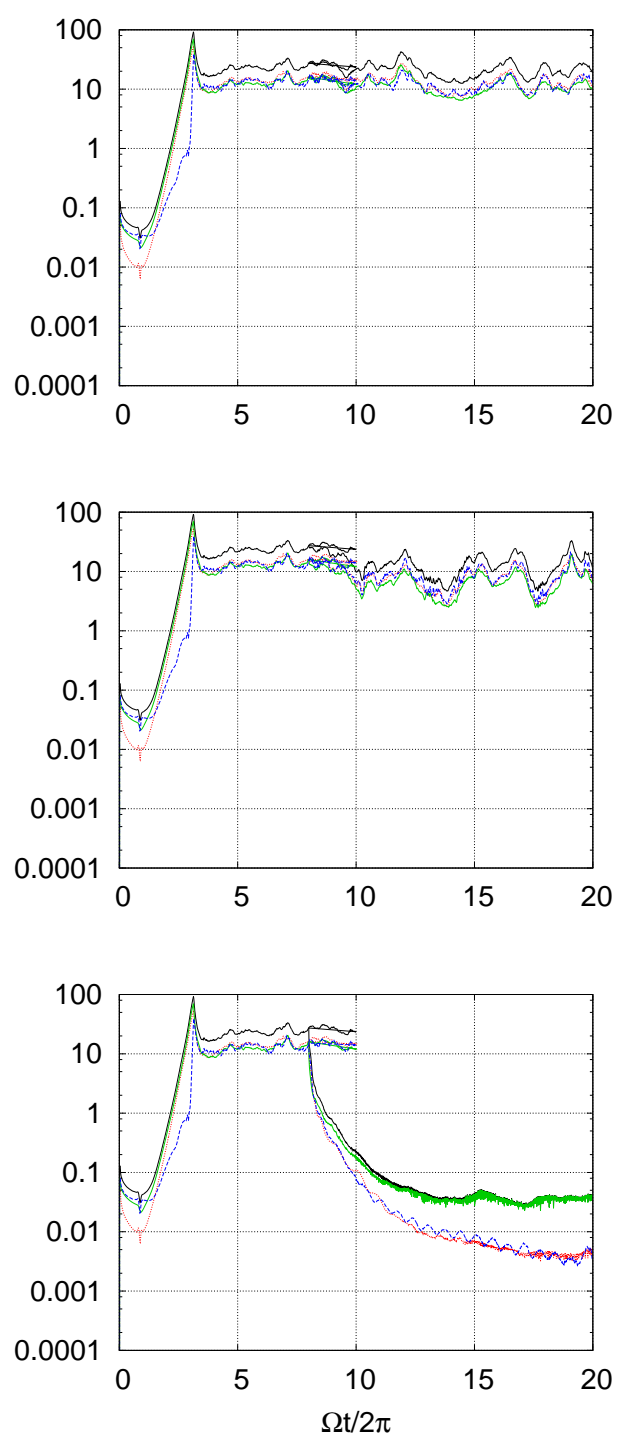

Fig. 2.- Time evolution of the averaged current density for three magnetic diffusivity models. Models are (a) $\beta=400, R_{M}=0.6, J_{\text {crit }} / J_{\text {eqp }}=1$ (b) $\beta=400, R_{M}=0.2, J_{\text {crit }} / J_{\text {eqp }}=1$ (c) $\beta=400, R_{M}=0.2$, $J_{\text {crit }} / J_{\text {eqp }}=10$. The graphs show typical current behavior for (a) $\bigcirc$ active zone, (b) $\triangle$ sustained zone, or (c) $\times$ dead zone. 

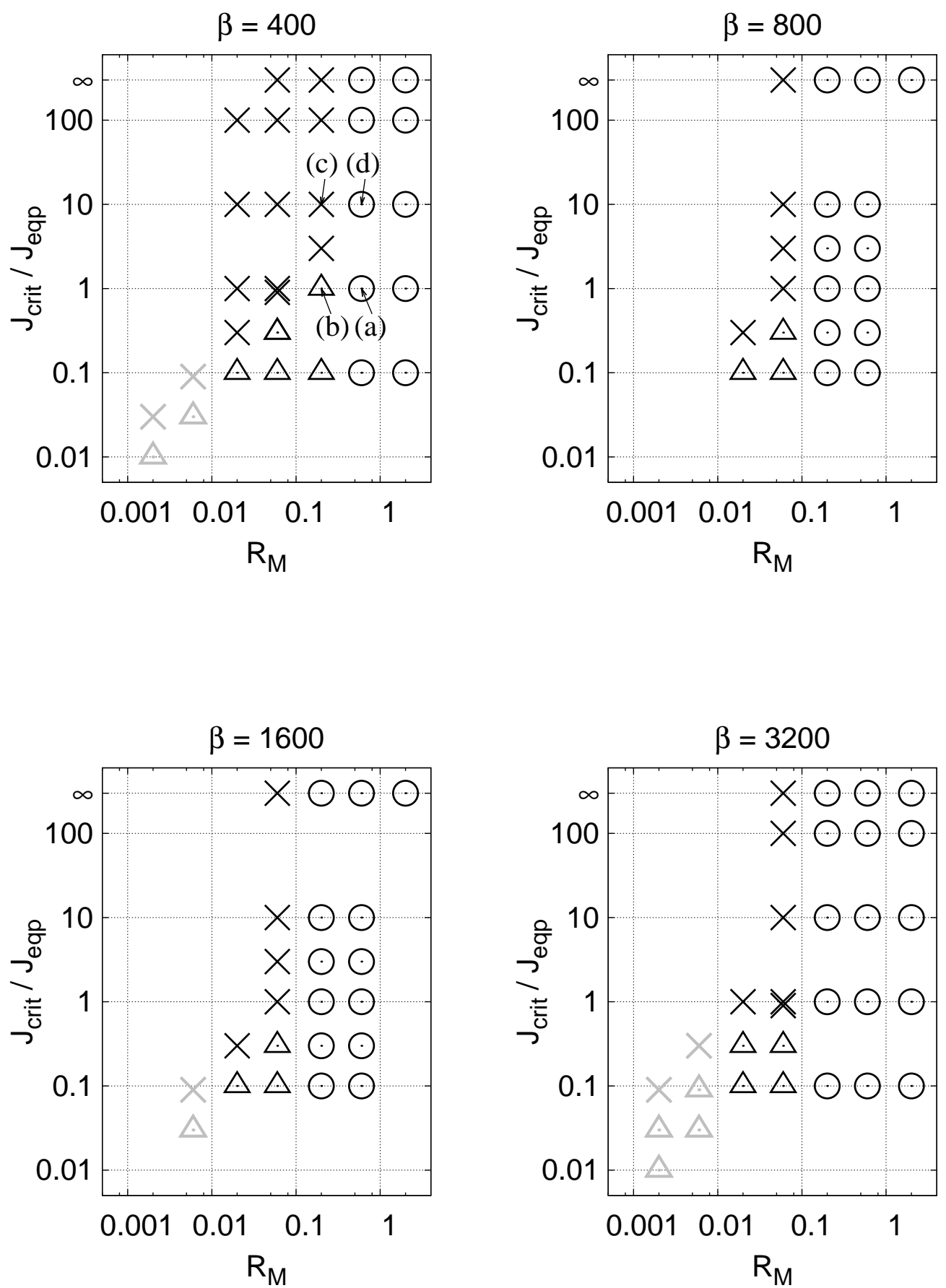

Fig. 3.- Distribution of the sets of parameters $\left(\beta, R_{\mathrm{M}}, J_{\text {crit }} / J_{\text {eqp }}\right)$ in our simulations. We classify each of them as either $\bigcirc$ active zone, $\triangle$ sustained zone, or $\times$ dead zone. This page includes the data for $400 \leq \beta \leq 3200$. The parameters classified by extrapolations are marked by light gray symbols. 

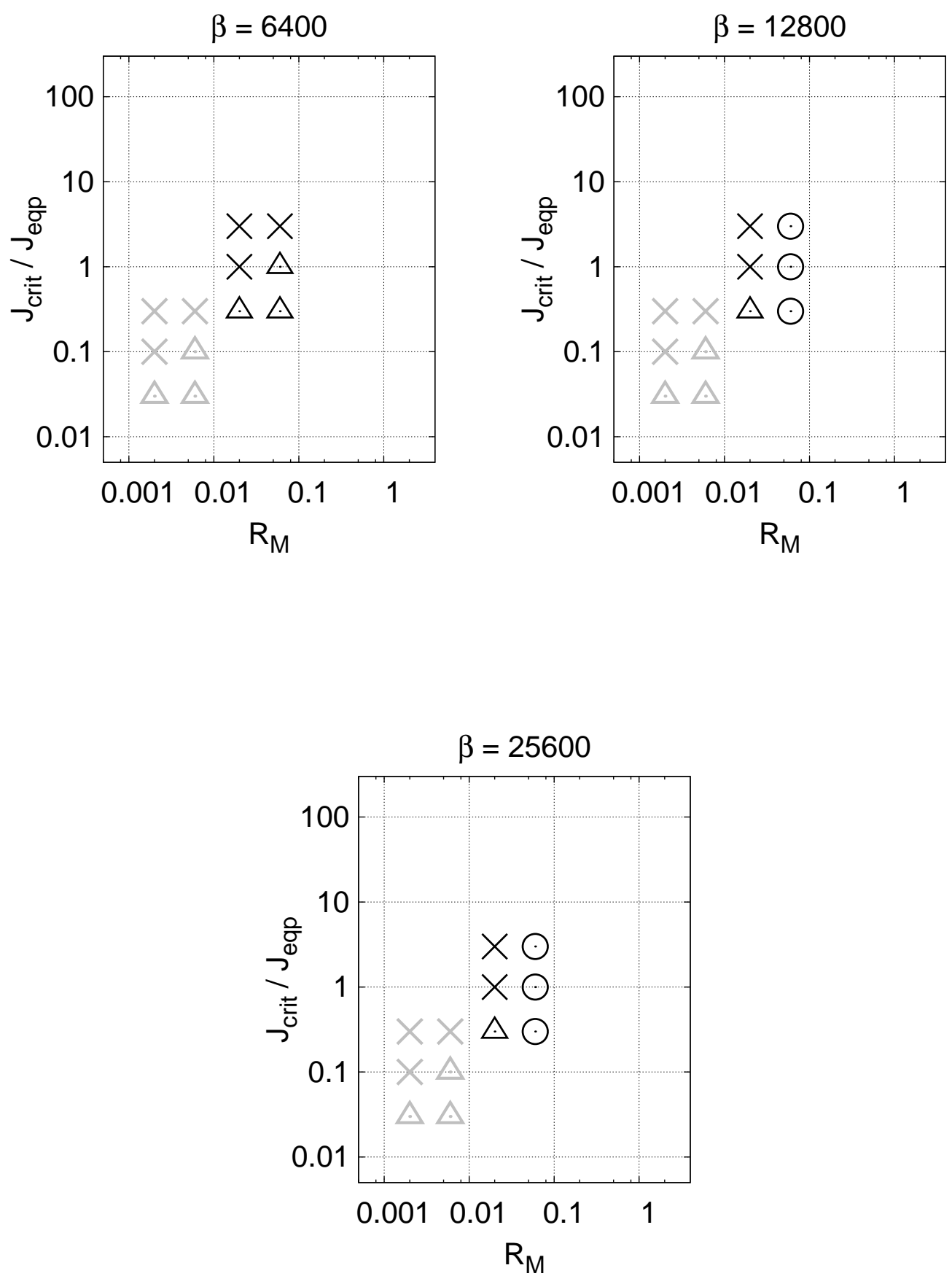

Fig. 4. - Continued from Figure 3, the data for $6400 \leq \beta \leq 25600$. 
restart from $t=0$

(a)

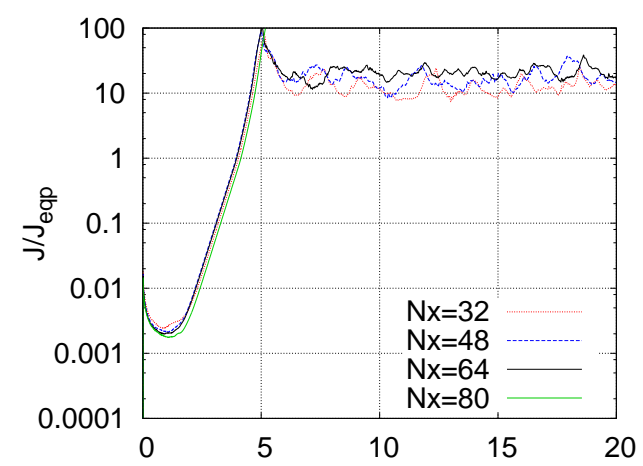

(b)

$\triangle$

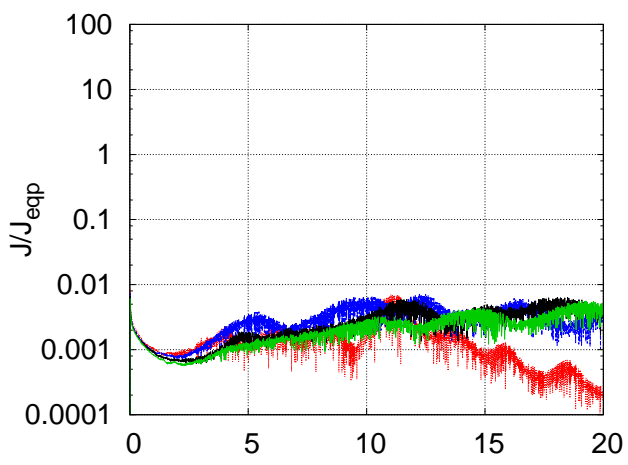

(c)

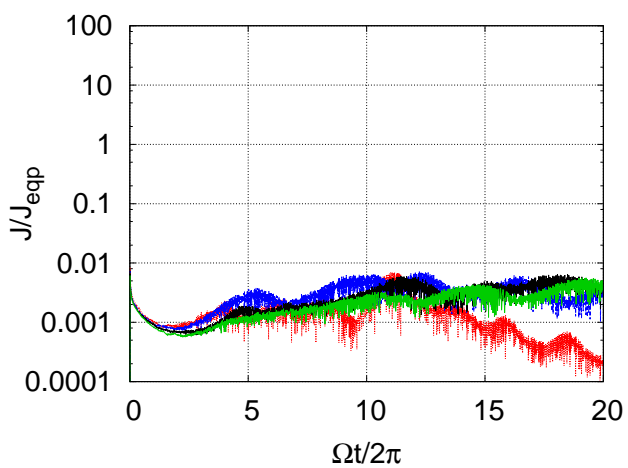

restart from $t=16 \pi / \Omega$
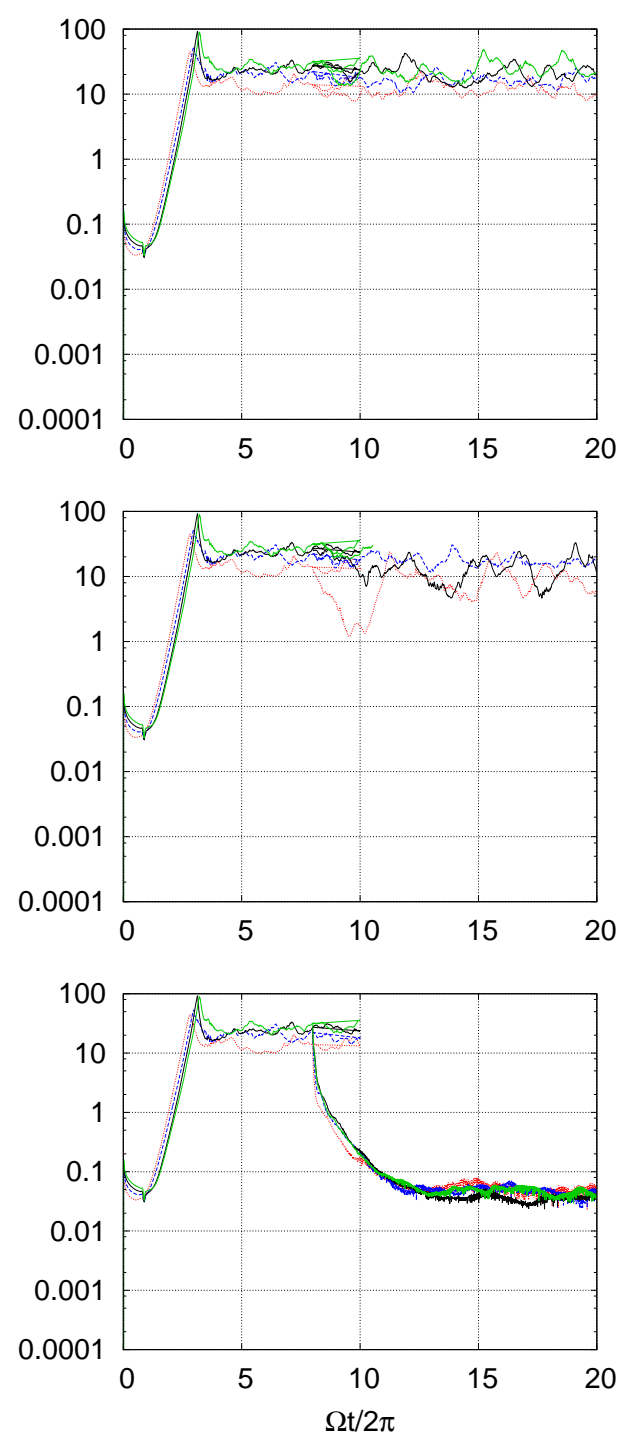

Fig. 5.- Dependence of the MRI behavior on the resolution. The development of electric current over time in the simulations using the different numerical resolution $N_{x}=32,48,64$ (fiducial) and 80 while keeping the aspect ratio $N_{x}: N_{y}: N_{z}=1: 2: 1$, for three different set of parameters (a) $\beta=400, R_{M}=0.6$, $J_{\text {crit }} / J_{\text {eqp }}=1$, (b) $\beta=400, R_{M}=0.2, J_{\text {crit }} / J_{\text {eqp }}=1$ and (c) $\beta=400, R_{M}=0.2, J_{\text {crit }} / J_{\text {eqp }}=10$. They are typical parameter sets for (a) $\bigcirc$ active zone, (b) $\triangle$ sustained zone, and (c) $\times$ dead zone, respectively. 
restart from $t=0$

(a)

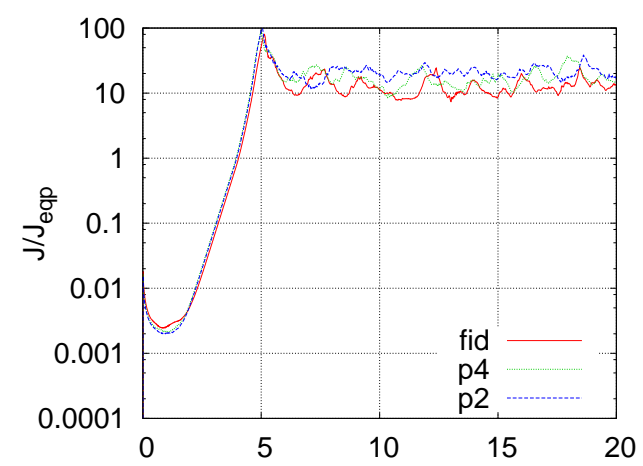

(b)

$\triangle$

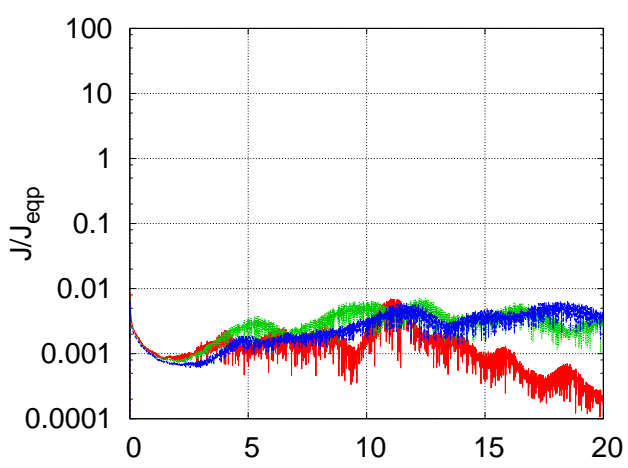

(c)

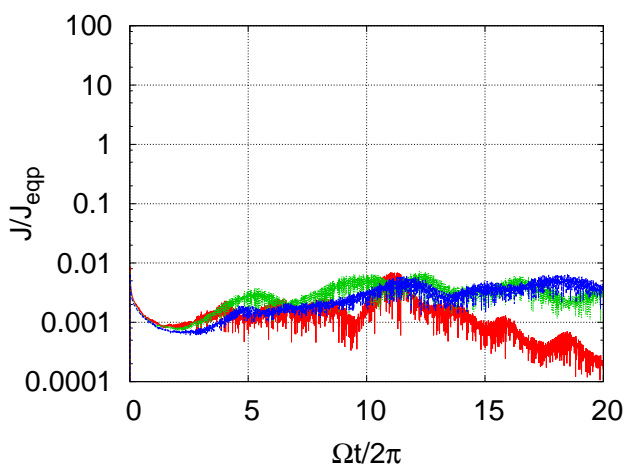

restart from $t=16 \pi / \Omega$
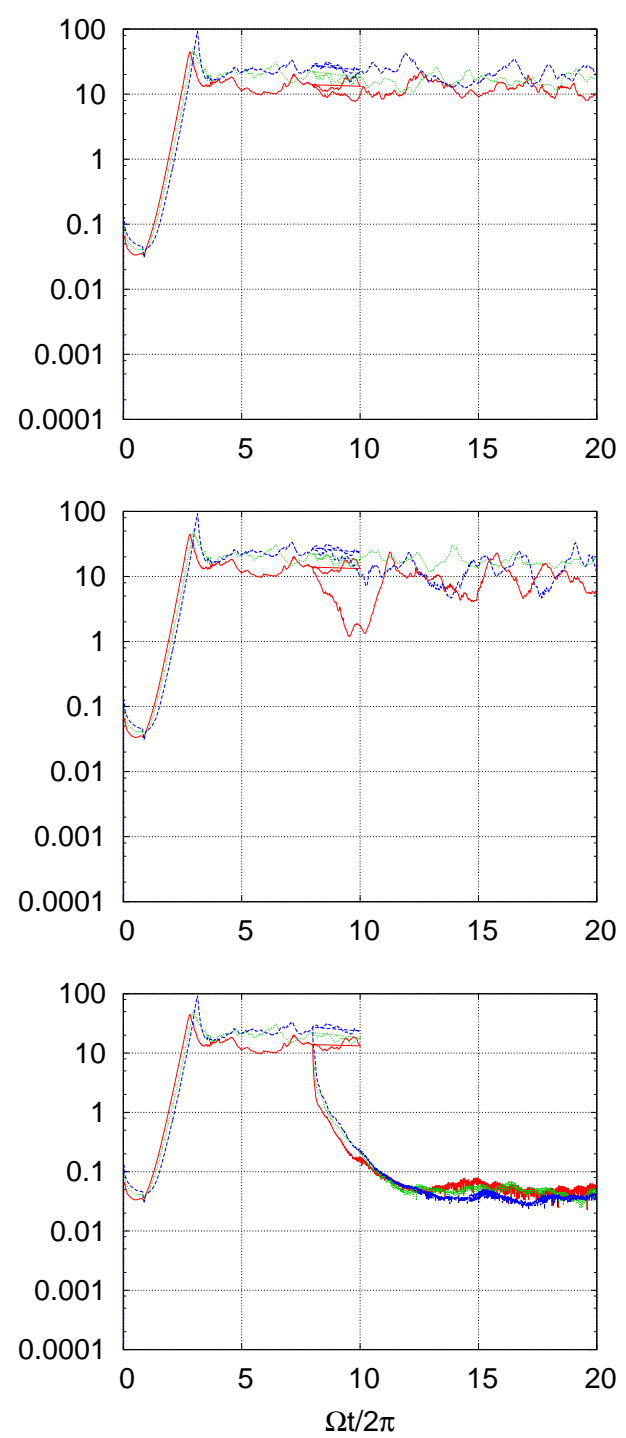

Fig. 6.- Dependence of the MRI behavior on resistivity models. The development of electric current over time in the simulations using different resistivity models fid( Eqs. (1) (2)), p2 (Eq. 11) and p4 (Eq. 12), for three sets of parameters (a) $\beta=400, R_{M}=0.6, J_{\text {crit }} / J_{\text {eqp }}=1$, (b) $\beta=400, R_{M}=0.2, J_{\text {crit }} / J_{\text {eqp }}=1$ and (c) $\beta=400, R_{M}=0.2, J_{\text {crit }} / J_{\text {eqp }}=10$; which are typical parameter sets for (a) $\bigcirc$ active zone, (b) $\triangle$ sustained zone, and $(\mathrm{c}) \times$ dead zone, respectively. 
cal resolutions, we have performed simulations using the different numerical resolution $N_{x}=$ $32,48,64$ (fiducial) and 80 while keeping the aspect ratio $N_{x}: N_{y}: N_{z}=1: 2: 1$, for three different set of parameters $\left(\beta, R_{M}, J_{\text {crit }} / J_{\text {eqp }}\right)=$ $(400,0.6,1),(400,0.2,1)$ and $(400,0.2,1)$. Figure 5 summarizes the convergence tests. Within these sets of parameters, we observe that our classification does not depend on the numerical resolution.

We have also studied if our result depends on the model of nonlinear Ohm's law. In addition to our fiducial(fid) model, Eq. (2), we have studied two smoothly transitting models, Eqs. (11) and (12). To distinguish the three models see Figure 1.

Figure 6 summarizes the time evolution of the current density in the simulations with the three typical sets of parameters $\left(\beta, R_{M}, J_{\text {crit }} / J_{\text {eqp }}\right)=$ $(400,0.6,1),(400,0.2,1)$ and $(400,0.2,1)$. Within the regime we have tested, the hysteresis behavior does not depend on the detail of the non-linear resistivity models.

From Figures 3 and 4 we can see the following condition for the $\triangle$ sustained zone:

$$
\frac{J_{\text {crit }}}{J_{\text {eqp }}} \frac{1}{R_{\mathrm{M}}} \leq f_{\mathrm{whb}},
$$

where $f_{\text {whb }}$ is a proportionality constant that satisfies $f_{\mathrm{whb}} \simeq 5-15$ for $400 \leq \beta \leq 1600$, and $f_{\text {whb }} \simeq 15-50$ for $\beta \geq 3200$. Hereafter, we interpret this in terms of the work-heat balance in the resistive MRI.

We also remark that within parameter regions that is in active zone, $R_{\mathrm{M}}<1$, and with larger $J_{\text {crit }}$, we observed the large-amplitude timevariability of physical quantities such as magnetic fields due to repeated growth and reconnection of the channel solutions. This phenomenon is reported by Fleming et al. (2000).

\subsection{Interpretation of The Simulation Re- sults}

In this section, we show that Equation (21) can be understood as a condition of the balance between the magnetic energy dissipated by Joule heating per unit volume $\left(W_{\mathrm{J}}\right)$ and the work done by shearing motion per unit volume $\left(W_{\mathrm{sh}}\right)$.

Let us define $Q_{\text {whb }}$ as the left hand side of Equa- tion (21):

$$
Q_{\mathrm{whb}} \equiv \frac{J_{\mathrm{crit}}}{J_{\mathrm{eqp}}} \frac{1}{R_{\mathrm{M}}} .
$$

The condition for self-sustained MRI is $Q_{\text {whb }}<$ $f_{\mathrm{whb}}$, which is to be explained in this section.

First, substitute $R_{\mathrm{M}} \equiv v_{\mathrm{Az}}{ }^{2} / \eta_{0} \Omega$;

$$
Q_{\mathrm{whb}}=\frac{J_{\mathrm{crit}}}{J_{\mathrm{eqp}}} \frac{\eta_{0} \Omega}{v_{\mathrm{Az}}{ }^{2}}
$$

Next, when the MRI is active, $\left\langle J^{2}\right\rangle^{1 / 2} \simeq$ $f_{\text {sat }} J_{\text {eqp }}$ where $f_{\text {sat }}$ is of the order of 10 . Since this average current strength lies in super-critical regime of Ohm's law $\left(J>J_{\text {crit }}\right)$, the electric field is $E_{\text {crit }}^{\prime}=4 \pi c^{-2} \eta_{0} J_{\text {crit }}$ as modeled in Equation (1) and (2). Therefore, Joule heating per unit volume is estimated as

$$
\begin{aligned}
W_{\mathrm{J}} & =E_{\text {crit }}^{\prime} \cdot\left\langle J^{2}\right\rangle^{1 / 2} \\
& =E_{\text {crit }}^{\prime} \cdot f_{\mathrm{sat}} J_{\mathrm{eqp}} \\
& =4 \pi f_{\mathrm{sat}} c^{-2} \eta_{0} J_{\mathrm{crit}} J_{\mathrm{eqp}}
\end{aligned}
$$

To explain the work-heat balance qualitatively, this estimate needs correction due to the high space variability of current field under the discharge conditions. We introduce $f_{\text {fill }}$, the filling factor, the ratio of the volume that contributes to the Joule heating to the total volume. Formally, $f_{\text {fill }}$ is defined as the ratio between volume averages of the actual Joule heat generated and the Joule heat estimated by this method:

$$
\begin{aligned}
f_{\text {fill }} & \equiv \frac{\left\langle E^{\prime} J\right\rangle}{E^{\prime}{ }_{\text {crit }} \cdot\left\langle J^{2}\right\rangle^{1 / 2}} \\
& =\frac{\langle f(J) J\rangle}{\left\langle J^{2}\right\rangle^{1 / 2}},
\end{aligned}
$$

where

$$
f(J)=\left\{\begin{array}{ccc}
1 & \text { if } & J>J_{\text {crit }} \\
\frac{J}{J_{\text {crit }}} & \text { if } & J<J_{\text {crit }}
\end{array} .\right.
$$

Using this $f_{\text {fill }}$, Equation (24) is rewritten as:

$$
W_{\mathrm{J}} \equiv 4 \pi f_{\text {fill }} f_{\text {sat }} c^{-2} \eta_{0} J_{\text {crit }} J_{\text {eqp }}
$$

Substituting $\eta_{0}$ in (23) with (27) gives

$$
\begin{aligned}
Q_{\mathrm{whb}} & =\frac{c^{2} W_{\mathrm{J}} \Omega}{4 \pi f_{\text {fill }} f_{\mathrm{sat}} J_{\mathrm{eqp}}{ }^{2} v_{\mathrm{Az}}{ }^{2}}, \\
W_{\mathrm{J}} & =\frac{4 \pi f_{\text {fill }} f_{\mathrm{sat}} J_{\mathrm{eqp}}{ }^{2} v_{\mathrm{Az}}{ }^{2} Q_{\mathrm{whb}}}{c^{2} \Omega} .
\end{aligned}
$$


Substituting $v_{\mathrm{Az}}$ with Equation (17) and then $c_{s}$ with (15), $J_{\text {eqp }}$ with Equation (14) and then $B_{\text {eqp }}$ with Equation (13) gives

$$
W_{\mathrm{J}}=\frac{4 f_{\text {fill }} f_{\mathrm{sat}} P_{0} \Omega Q_{\mathrm{whb}}}{\beta} .
$$

On the other hand,

$$
\begin{aligned}
W_{\mathrm{sh}} & \equiv \frac{3}{2} \Omega\left\langle w_{x y}\right\rangle \\
& =\frac{3}{2} \alpha \Omega P
\end{aligned}
$$

where $\alpha$ is Shakura \& Sunyaev (1973) 's $\alpha$ parameter. Substituting Equation (30) into Equation (29), one obtains

$$
W_{\mathrm{J}}=\frac{8 f_{\mathrm{fill}} f_{\mathrm{sat}} Q_{\mathrm{whb}}}{3 \alpha \beta} W_{\mathrm{sh}}
$$

For the MRI to sustain itself by the discharge process, the Joule heating $W_{\mathrm{J}}$ needs to be equal or smaller than $W_{\mathrm{sh}}$ :

$$
W_{\mathrm{J}} \lesssim W_{\mathrm{sh}} .
$$

Therefore, we have the following constraint on the left hand side of Equation (23):

$$
\begin{aligned}
Q_{\mathrm{whb}} & =\frac{3 \alpha \beta}{8 f_{\text {fill }} f_{\mathrm{sat}}} \frac{W_{\mathrm{J}}}{W_{\mathrm{sh}}}, \\
\frac{J_{\text {crit }}}{J_{\text {eqp }}} \frac{1}{R_{\mathrm{M}}} & \lesssim \frac{3 \alpha \beta}{8 f_{\text {fill }} f_{\mathrm{sat}}} \equiv f_{\mathrm{whb}}(\beta) .
\end{aligned}
$$

Thus, the work-heat balance poses an upper limit on the product of $J_{\text {crit }} / J_{\text {eqp }}$ and $1 / R_{\mathrm{M}}$, provided that $f_{\text {fill }}, f_{\text {sat }}$ and $\alpha$ are constants that do not depend on $J_{\text {crit }}$ and $R_{\mathrm{M}}$, but only on $\beta$. This explains the inverse-proportional relations observed in Figures 3 and 4 . The $f_{\text {whb }}(\beta)$ calculated with this interpretation using the experimental data are in Table 3.

We can further simplify Equation (33) by using the saturation predictor proposed by HGB95. The proposed predictors read

$$
\begin{aligned}
\alpha P & =0.61 \pm 0.06 \overline{\left\langle\frac{B^{2}}{8 \pi}\right\rangle} \\
\overline{\left\langle\frac{B^{2}}{8 \pi}\right\rangle} & =(1.21 \pm 0.29) \cdot 2 \pi \sqrt{\frac{16}{15} \cdot \frac{2}{\beta}} P_{0} .(35)
\end{aligned}
$$

Using this, Equation (33) is rewritten as follows:

$$
\begin{aligned}
\frac{J_{\text {crit }}}{J_{\text {eqp }}} \frac{1}{R_{\mathrm{M}}} & \lesssim f_{\text {whb }}(\beta) \\
& \simeq(2.54 \pm 0.66) \frac{\beta^{1 / 2}}{f_{\text {fill }} f_{\text {sat }}} .
\end{aligned}
$$

By ignoring the dependence of $f_{\text {fill }}$ and $f_{\text {sat }}$ on $\beta$, we assume $\overline{f_{\text {fill }}}=0.264 \pm 0.007$ and $\overline{f_{\text {sat }}}=13.1 \pm$ 3.1. This further simplifies the Equation (33) as:

$$
f_{\mathrm{whb}}(\beta) \simeq(0.74 \pm 0.26) \beta^{1 / 2} .
$$

This is in agreement with our experimental data (Table 3) within factor of 2.

\section{Distribution of The Three MRI Zones within The Protoplanetary Disks}

\subsection{Protoplanetary Disk Model}

In the previous section, we performed the shearing-box simulations of MRI with nonlinear Ohm's law, and found the three classes of MRI behavior; we named them active, dead and sustained zones. We also found the condition for the MRI to be self-sustained. In this section, we apply the findings to the global model of the protoplanetary disks and analyze how they are divided into the three zones.

We use Minimum-Mass Solar Nebula (MMSN) model introduced by Hayashi et al. (1985) as the fiducial disk model;

$$
\begin{gathered}
\Sigma=f_{\Sigma} \Sigma_{0}\left(\frac{r}{\mathrm{AU}}\right)^{-q}, \\
T=T_{0}\left(\frac{r}{\mathrm{AU}}\right)^{-\frac{1}{2}} .
\end{gathered}
$$

Here, $\Sigma_{0}=1.7 \times 10^{3} \mathrm{~g} \mathrm{~cm}^{-3}$ and $T_{0}=2.8 \times$ $10^{2} \mathrm{~K}$ are the surface density and the temperature at $1 \mathrm{AU}$, respectively. $f_{\Sigma}$ is the nondimensional surface density parameter. Fiducial value for the surface density power index is $q=3 / 2$. Since we assume the isotropic equation of state (EOS), the ratio of specific heats $\gamma=1$ in our model, and the thermal velocities for gas molecules and electrons are

$$
\begin{aligned}
& c_{s}(r)=\sqrt{\frac{k_{B} T}{\mu m_{H}}}, \\
& v_{e}(r)=\sqrt{\frac{k_{B} T}{m_{e}}},
\end{aligned}
$$




\begin{tabular}{c|cccc|c}
$\beta$ & $\bar{\alpha}$ & $\overline{\left\langle\frac{B^{2}}{8 \pi P_{0}}\right\rangle}$ & $\overline{f_{\text {sat }}}$ & $\overline{f_{\text {fill }}}$ & $f_{\text {whb }}(\beta)$ \\
\hline 400 & $0.176 \pm 0.036$ & $0.222 \pm 0.040$ & $14.5 \pm 1.7$ & $0.252 \pm 0.001$ & $7.19 \pm 0.81$ \\
800 & $0.143 \pm 0.035$ & $0.206 \pm 0.057$ & $17.4 \pm 2.7$ & $0.259 \pm 0.001$ & $9.33 \pm 1.03$ \\
1600 & $0.104 \pm 0.027$ & $0.166 \pm 0.047$ & $17.4 \pm 1.8$ & $0.265 \pm 0.002$ & $13.3 \pm 2.3$ \\
3200 & $0.0523 \pm 0.0206$ & $0.0916 \pm 0.0411$ & $12.2 \pm 3.9$ & $0.262 \pm 0.008$ & $19.0 \pm 3.9$ \\
6400 & $0.0236 \pm 0.0041$ & $0.0386 \pm 0.0072$ & $9.58 \pm 1.04$ & $0.263 \pm 0.003$ & $22.2 \pm 1.6$ \\
12800 & $0.0182 \pm 0.0032$ & $0.0300 \pm 0.0060$ & $9.84 \pm 0.88$ & $0.270 \pm 0.000$ & $32.6 \pm 2.8$ \\
25600 & $0.0185 \pm 0.0063$ & $0.0315 \pm 0.0128$ & $10.7 \pm 1.5$ & $0.275 \pm 0.001$ & $58.5 \pm 12.4$
\end{tabular}

Table 3: The $f_{\mathrm{whb}}(\beta)$ calculated from the experimental data. We first calculated the time and space averaged quantities $\bar{\alpha}$ and $\overline{f_{\text {sat }}}$ for each runs. Then for the ensemble of runs, we calculated the means and the standard deviations of the quantities. The ensemble constitutes of runs that (1) belong to $\triangle$ sustained zone, (2) are restarted runs $(t=16 \pi / \Omega, 18 \pi / \Omega, 20 \pi / \Omega)$ so that they are magnetorotationally unstable, and (3) have the largest product $J_{\text {crit }} / J_{\text {eqp }} \cdot 1 / R_{\mathrm{M}}$ so that they face the $\triangle$ sustained zone - $\times$ dead zone boundary.

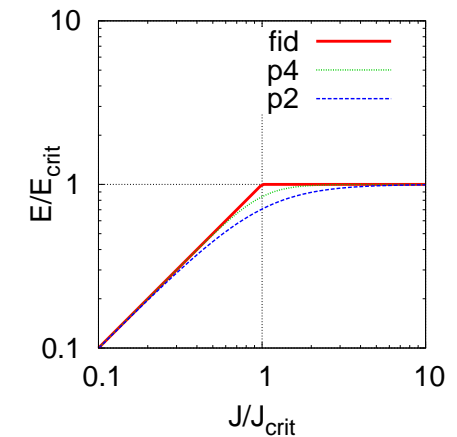

Fig. 1.- Electric field amplitude as functions of current in the three different models of nonlinear Ohm's law. The symbols fid, p2, and p4 correspond to equation (2), (11), and (12),respectively. respectively, where $\mu$ is the mean molecular weight of the gas.

Since we neglect the self gravity of the disk, the disk is in Kepler rotation and its orbital angular velocity is

$$
\Omega(r)=\sqrt{\frac{G M_{*}}{r^{3}}} .
$$

From the equilibrium between vertical pressure gradient and vertical component of the stellar gravity, the disk density and pressure distributions are:

$$
\begin{aligned}
\rho(r, z) & =\frac{\Sigma}{\sqrt{2 \pi} H} \exp \left(\frac{-z^{2}}{2 H^{2}}\right), \\
P(r, z) & =\frac{\rho k_{B} T}{\mu m_{H}}
\end{aligned}
$$

where $H$ is the definition of the disk scale-height in this paper c.f. Equation (15).

For simplicity, we assume that the every dust particle to be solid sphere of the equal radius $a_{d}$ and density $\rho_{s}$. The mass $m_{d}$ and geometrical cross section $\sigma_{d}$ of the dust particle are

$$
\begin{aligned}
m_{d} & =\frac{4 \pi}{3} \rho_{s} a_{d}^{3}, \\
\sigma_{d} & =\pi a_{d}^{2},
\end{aligned}
$$

respectively. The fiducial values are $a_{d}=0.1 \mu \mathrm{m}$ and $\rho_{s}=3 \mathrm{~g} \mathrm{~cm}^{-3}$.

Using this $m_{d}$ and dust-to-gas density ratio $f_{d}=0.01$, the number densities of dust and gas 
component are:

$$
\begin{aligned}
n_{g}(r, z) & =\frac{\rho}{\mu m_{H}}, \\
n_{d}(r, z) & =\frac{f_{d} \rho}{m_{d}} .
\end{aligned}
$$

\subsection{Ionization Processes}

Methods for calculating the charge equilibrium of the dust-plasma in the protoplanetary disks has been studied (Umebayashi \& Nakano 1980, 2009; Fujii et al. 2011). Among those we use O09's method because of its numerical efficiency and generality. First, the gas column density above $\left(\chi_{\top}\right)$ and below $\left(\chi_{\perp}\right)$ the certain coordinate $(r, z)$ in the disk are

$$
\begin{aligned}
\chi_{\top}(r, z) & =\int_{z}^{\infty} \rho d z \\
& =\frac{\Sigma}{2}\left[1-\operatorname{erf}\left(\frac{z}{\sqrt{2} H}\right)\right], \\
\chi_{\perp}(r, z) & =\int_{-\infty}^{z} \rho d z \\
& =\frac{\Sigma}{2}\left[1+\operatorname{erf}\left(\frac{z}{\sqrt{2} H}\right)\right],
\end{aligned}
$$

respectively, where

$$
\operatorname{erf}(x) \equiv \frac{2}{\sqrt{\pi}} \int_{x}^{\infty} e^{-t^{2}} d t
$$

is the error function.

According to SMUN00, the effective ionization rate in the disk is

$$
\begin{aligned}
\zeta(r, z) & \approx \frac{\zeta_{\mathrm{CR}}}{2}\left\{\exp \left(-\frac{\chi_{\mathrm{T}}}{\chi_{\mathrm{CR}}}\right)\right. \\
& \left.+\exp \left(-\frac{\chi_{\perp}}{\chi_{\mathrm{CR}}}\right)\right\}+\zeta_{\mathrm{RA}}
\end{aligned}
$$

where $\chi_{\mathrm{CR}}=96 \mathrm{~g} \mathrm{~cm}^{-2}, \zeta_{\mathrm{CR}}=1.0 \times 10^{-17} \mathrm{~s}^{-1}$, and $\zeta_{\mathrm{RA}}=6.9 \times 10^{-23} \mathrm{~s}^{-1}$.

Using this, O09's nondimensional parameter $\Theta$ is calculated as

$$
\Theta=\frac{\zeta n_{g} e^{2}}{s_{i} c_{s} \sigma_{d} a_{d} n_{d}^{2} k_{B} T},
$$

and $\Gamma$ is defined as the solution of the equation

$$
\frac{1}{1+\Gamma}-\left(\frac{s_{i}}{s_{e}} \sqrt{\frac{m_{e}}{\mu m_{H}}} \exp \Gamma+\frac{\Gamma}{\Theta}\right)=0 .
$$

Once Equation (56) is numerically solved for $\Gamma$, we can calculate the number density of ions and electrons, $n_{i}, n_{e}$, as well as the root mean square of the charge per dust particle, $\sqrt{\left\langle Z^{2}\right\rangle} e$, as:

$$
\begin{aligned}
n_{i}(r, z) & =\frac{\zeta n_{g}}{s_{i} c_{s} \sigma_{d} n_{d}(1+\Gamma)}, \\
n_{e}(r, z) & =\frac{\zeta n_{g} \exp \Gamma}{s_{e} v_{e} \sigma_{d} n_{d}}, \\
\left\langle Z^{2}\right\rangle & =\left(\frac{\Gamma a_{d}}{\lambda}\right)^{2}+\frac{1+\Gamma}{2+\Gamma} \frac{a_{d}}{\lambda} \\
\text { where } \lambda & =\frac{e^{2}}{k_{B} T},
\end{aligned}
$$

We assume sticking probabilities $s_{i}=1$ and $s_{e}=$ 0.3 as assumed by O09.

Next, we estimate the plasma conductivity using the method of SMUN00. The rate coefficient for the collision between the neutrals and the ions is

$$
\langle\sigma v\rangle_{i}=2.41 \pi\left(\frac{\alpha e^{2}}{\mu m_{H}}\right)^{\frac{1}{2}}
$$

We use $\alpha=7.66 \times 10^{-25} \mathrm{~cm}^{3}$ as an averaged polarizability. $\langle\sigma v\rangle_{e}$ is the rate coefficient for collision between neutrals and electron, whose form is given in SMUN00. The rate coefficient for dust particles is

$$
\langle\sigma v\rangle_{d}=\frac{4 \pi}{3} a_{d}^{2} c_{s}
$$

This expression is valid as long as $a_{d}$ is much smaller than the mean free path of the gas molecules.

With these, the magnetic diffusivity is calculated component-wise:

$$
\begin{aligned}
\eta_{e} & =\frac{c^{2} m_{e} n_{g}\langle\sigma v\rangle_{e}}{4 \pi e^{2} n_{e}}, \\
\eta_{i} & =\frac{c^{2} \mu m_{H} n_{g}\langle\sigma v\rangle_{i}}{4 \pi e^{2} n_{i}}, \\
\eta_{d} & =\frac{c^{2} \mu m_{H} n_{g}\langle\sigma v\rangle_{d}}{4 \pi\left\langle Z^{2}\right\rangle e^{2} n_{d}}, \\
\eta_{0} & =\left(\eta_{e}{ }^{-1}+\eta_{i}{ }^{-1}+\eta_{d}{ }^{-1}\right)^{-1}
\end{aligned}
$$

The value of $E^{\prime}$ crit is set by the condition that the kinetic energy of the electrons accelerated by the electric field is large enough to initiate the electron 
avalanche.

$$
\begin{aligned}
\Delta W & =f_{\mathrm{DP}} e E^{\prime}{ }_{\text {crit }} l_{\mathrm{mfp}} \\
E_{\text {crit }}^{\prime} & =\frac{\Delta W}{f_{\mathrm{DP}} e l_{\mathrm{mfp}}} \\
& =\frac{\Delta W n_{g}\langle\sigma v\rangle_{e}}{f_{\mathrm{DP}} e v_{e}}
\end{aligned}
$$

Here, $f_{\mathrm{DP}}=0.43 \sqrt{\mu m_{H} / m_{e}}$ is the coefficient for average energy of electrons in weakly ionized plasma (IS05), and $l_{\mathrm{mfp}}=v_{e} /\left(n_{g}\langle\sigma v\rangle_{e}\right)$ is the mean free path of electrons. For ionization energy we use the value for a hydrogen molecule $\Delta W=15.4 \mathrm{eV}$.

With this, the critical current is

$$
J_{\text {crit }}=\frac{c^{2}}{4 \pi \eta_{0}} E^{\prime} \text { crit } .
$$

Note that the discharge electric field, Equation (66) is calculated using the strong electric field limit of the electron distribution function, while we used the weak field limit formulae for charge distributions, Equations (55)-(61). In this paper we adopt this treatment for simplicity. A more consistent treatment will be the topic of another paper in preparation.

\subsection{The Self-Sustained MRI in Global Disk Models}

Now we study the distribution of active, sustained and dead zones in the protoplanetary disk models. SMUN00 gives the condition for MRI unstable region as follows:

$$
\frac{2 \pi v_{\mathrm{Az}}}{\Omega} \leq \sqrt{2} H \bigwedge \frac{2 \pi \eta}{v_{\mathrm{Az}}} \leq \sqrt{2} H .
$$

Combination of this with work-heat balance model Equation (21) gives the following conditions for active, sustained and dead zones, respectively:

$$
\begin{aligned}
& \frac{2 \pi v_{\mathrm{Az}}}{\Omega} \leq \sqrt{2} H \bigwedge \frac{2 \pi \eta}{v_{\mathrm{Az}}} \leq \sqrt{2} H \\
& \frac{2 \pi v_{\mathrm{AZ}}}{\Omega} \leq \sqrt{2} H \bigwedge \frac{2 \pi \eta}{v_{\mathrm{Az}}}>\sqrt{2} H \bigwedge \frac{J_{\text {crit }}}{J_{\text {eqp }}} \frac{1}{R_{\mathrm{M}}} \leq f_{\mathrm{whb}}
\end{aligned}
$$

$$
\frac{2 \pi v_{\mathrm{Az}}}{\Omega}>\sqrt{2} H \bigvee\left(\frac{2 \pi \eta}{v_{\mathrm{Az}}}>\sqrt{2} H \bigwedge \frac{J_{\text {crit }}}{J_{\text {eqp }}} \frac{1}{R_{\mathrm{M}}}>f_{\mathrm{whb}}\right)
$$

Using these Equations (69)-(71), we plot the active, sustained and dead zones for various global disk model Equations (39)-(40).

First, Figure 7 shows the unstable zones for varying disk surface density, $f_{\Sigma}=0.3, f_{\Sigma}=1$ (the fiducial model), $f_{\Sigma}=3$, and $f_{\Sigma}=10$. In this figure and following figures, the thick curves are the boundary of the work-heat balance model Equation (21), while the thin curves are the boundary of the instability condition in the resistive limit, i.e. the second condition in Equation (68). The solid and dashed curves correspond to the plasma beta at the mid-plane $\beta=100$ and $\beta=1000$, respectively. The active zones are marked by meshes, and the sustained zones are marked by horizontal stripes.

Figure 8 shows the active and sustained zones for dust-to-gas ratio $f_{d}=1, f_{d}=0.1, f_{d}=0.01$ (the fiducial model), and $f_{d}=10^{-4}$. The workheat balance condition Equation (21) is not affected by changing the dust properties such as dust-to-gas ratio $f_{d}$ or dust size $a_{d}$. One can understand this by rewriting the condition Equation (23) in the following form:

$$
\frac{E_{\text {crit }}^{\prime} \Omega}{4 \pi c^{-2} J_{\text {eqp }} v_{\mathrm{Az}}^{2}} \leq f_{\mathrm{whb}} .
$$

This form does not include a term affected by the dust properties, such as the magnetic diffusivity. On the other hand, $E^{\prime}{ }_{\text {crit }}$ is inversely proportional to the electron mean free path $l_{\mathrm{mfp}}$, and is proportional to the gas number density.

In Figure 9 we study the evolution of the active and sustained zones as the gas density becomes lower while the dust density is kept constant. We change the set $\left(f_{\Sigma}, f_{d}\right)$ from $(1,0.01)$ (the fiducial model $)$ to $(0.1,0.1),(0.01,1)$, and $\left(10^{-4}, 100\right)$. In Figure 9 zones are marked for $\beta=1000$. The midplane of the disk between the radii $2 \mathrm{AU}-20 \mathrm{AU}$ becomes the sustained zone as the gas density becomes $10^{-4}$ times the fiducial model.

\section{Conclusions and Discussions}

By performing numerical simulations of MHD with nonlinear Ohm's law of the three-dimensional local disks, we found hysteresis behavior for certain diffusivity model: If we start from the laminar-flow initial conditions with small seed fluctuations, the MRI does not activate because 

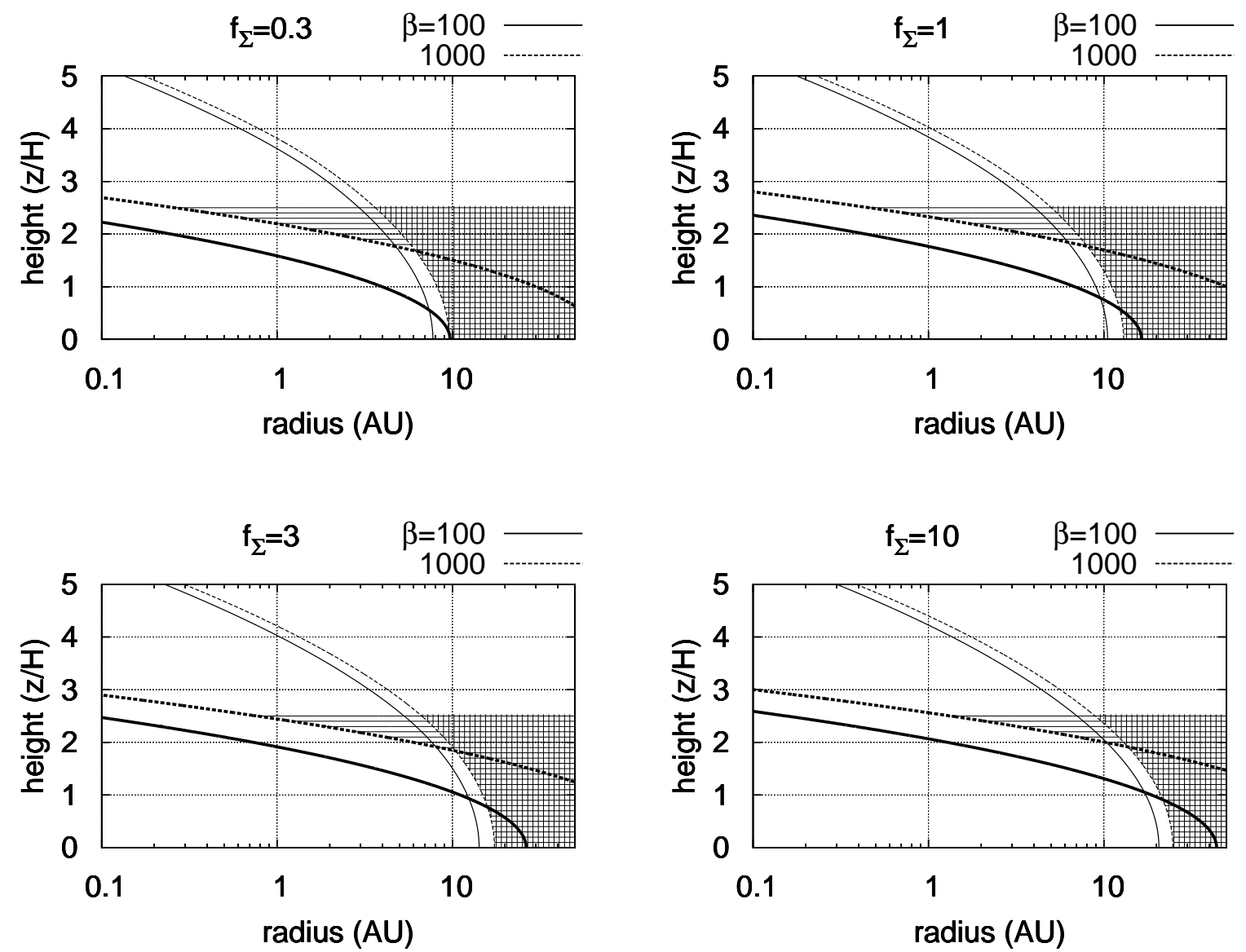

Fig. 7.- Unstable regions in the protoplanetary disks. The thin solid and thin dashed curves represent $\lambda_{\text {res }} / \sqrt{2} H=1$ for the cases of the magnetic field strength $\beta=100$ and 1000, respectively, inside of which is dead zone if the MRI self-sustainment is not taken into account (SMUN00). The regions above the thick solid and thick dashed curves satisfies Equation (21) for $\beta=100$ and 1000, respectively, and are sustained zones according to the work-heat balance model. We compare the unstable region predicted by SMUN00 to that predicted by our model for $\beta=1000$. The unstable regions according to SMUN00's and our model are marked by vertical and horizontal stripes, respectively. 

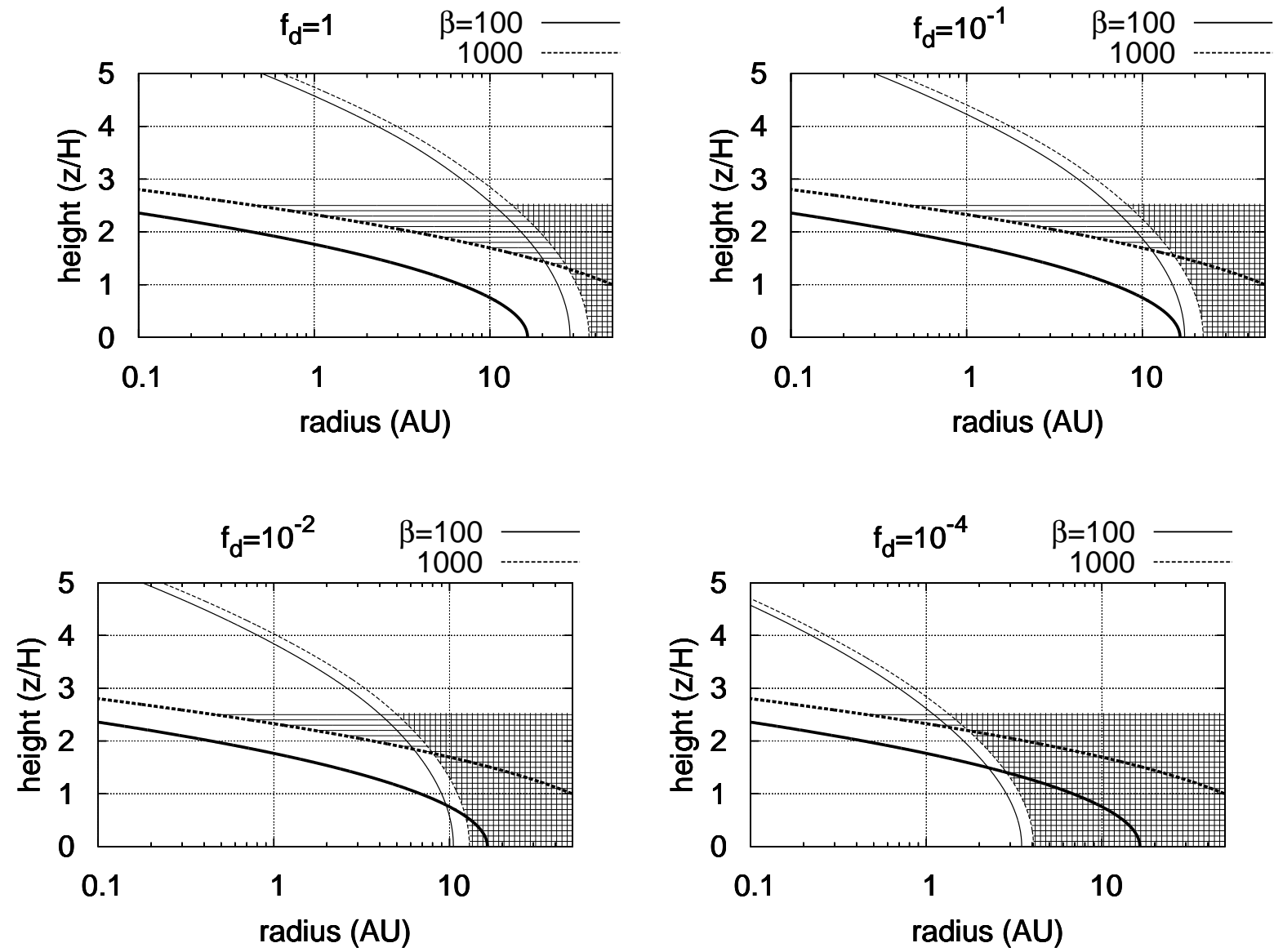

Fig. 8. - Unstable regions for different dust-to-gas ratio. The MRI-unstable region according to (SMUN00) and our model are marked by vertical and horizontal stripes, respectively, for $\beta=1000$. 

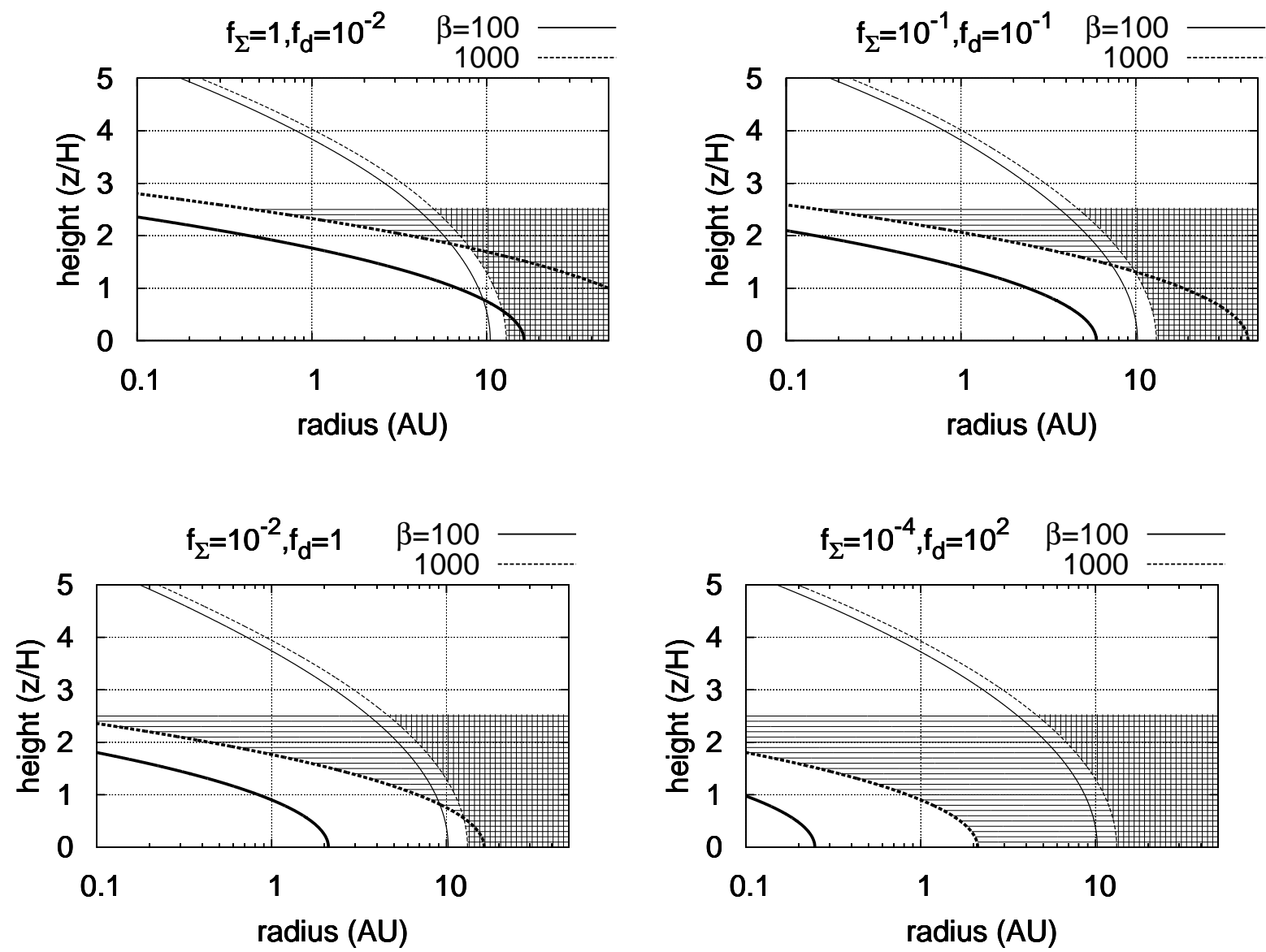

Fig. 9.- Change of unstable regions as the gas density of the disk decreases, while the dust density is kept constant. The MRI-unstable region according to (SMUN00) and our model are marked by vertical and horizontal stripes, respectively, for $\beta=1000$. 
of the diffusivity and the flow remains laminar; on the other hand, if we take MRI-turbulent state from an ideal-MHD simulation as initial conditions, MRI remains active under the same diffusivity model. We have surveyed in threeparameter space $\left(\beta, J_{\text {crit }}, R_{\mathrm{M}}\right)$ in search for the regions the self-sustained MRI in the context of IS05 takes place. We found the condition, the work-heat balance model, for this hysteresis behavior to take place. The model is $W_{\mathrm{J}} \lesssim W_{\mathrm{sh}}$, where $W_{\mathrm{J}}$ is the magnetic energy dissipated by Joule heating per unit volume and $W_{\text {sh }}$ is the work done by background shearing motion per unit volume. This leads to the proportionality relation $\frac{J_{\text {crit }}}{J_{\text {eqp }}} \frac{1}{R_{\mathrm{M}}} \leq f_{\text {whb }}$.

IS05 concluded that the the energy supply from the shearing motion should be $\sim 30$ times greater than the energy needed to supplying the enough ionization for the MRI, and predicted the entire disk to be active. However, applying the workheat balance model to various protoplanetary disk models, we have found that in most of the models, the sustained zone is above $z / H>2-3$.

We conclude that in the fiducial protoplanetary disks environment the Joule heating (which has been neglected in IS05) becomes the dominant energy dissipation channel and constrains the selfsustainment of MRI, and the midplane of the disk remain dead. However, the gas of the disk dissipates (Alexander et al. (2006a,b); Suzuki et al. (2010)) with observed timescale of $10^{6}-10^{7}$ years (Cieza et al. (2007); Hernndez et al. (2008)), while planetesimals remain and continue planet formation processes. In such late phase of the disk, the sustained zone occupies larger volume of the disk.

Although our nonlinear Ohm's law model is inspired by the lightning phenomena, whether the Joule heating in this model takes the form of spatially and temporally concentrated stream of ionizing electrons - lightning — or not, has yet to be studied in future works, employing nonthermal plasma studies. We limit ourselves to pointing out a few distinguishing properties of such lightning which makes it an interesting subject. First, the work-heat balance model suggests that in sustained zones the major portion of the shearing motion energy is converted to lightning. This means that the lightning is one of the most dominant energy channel in the sustained zones. It will also pose a significant back-reaction to the accretion dynamics. We will need to reconsider the contribution of lightning in situations it has been neglected due to lack of energy, such as in chondrule formation (Weidenschilling 1997).

The second point is related to the redox environment the lightning creates. Lightning induced by collisional charging of water ice dust overcomes the energetics problem (Muranushi 2010), but if applied as the chondrule heating source, it suffers from the redox environment mismatch. Water vapor creates oxidizing environment (Clayton et al. 1981; Rubin 2005) whereas major population of chondrules are considered to have formed in reducing environment (Lofgren 1989; Connolly et al. 1994; Jones \& Danielson 1997). However, the lightning in sustained zone proposed by IS05 and studied in this paper, is a result of pure MHD process, and thus is redox-neutral. Therefore, it can potentially explain chondrule heating in both reducing and oxidizing environment.

T.M. thanks Yuichiro Sekiguchi and Masaru Shibata for useful discussions. We thank the referee for the valuable comments. The simulations for this paper has been performed on computer cluster at Kyoto University built by T.M, and TSUBAME2.0 Grid Cluster at Tokyo Institute of Technology. The use of TSUBAME2.0 in this project was supported by JHPCN through its program "Joint Usage/Research Center for Interdisciplinary Large-scale Information Infrastructures." This work is supported by Grants-in-Aid from the Ministry of Education, Culture, Sports, Science, and Technology (MEXT) of Japan, No. 24103506 (T.M.), No. $22 \cdot 7006$ (S.O.), No. 18540238, No. 23244027, and No. 23103005 (S.I.).

\section{REFERENCES}

Alexander, R. D., Clarke, C. J., \& Pringle, J. E. 2006a, Monthly Notices of the Royal Astronomical Society, 369, 216

- 2006b, Monthly Notices of the Royal Astronomical Society, 369, 229

Balbus, S. A., \& Hawley, J. F. 1998, Reviews of Modern Physics, 70, 1

Blum, J., \& Wurm, G. 2008, Annual Review of Astronomy and Astrophysics, 46, 21 
Brauer, F., Henning, T., \& Dullemond, C. P. 2008, Astronomy and Astrophysics, 487, L1

Cieza, L., Padgett, D. L., Stapelfeldt, K. R., Augereau, J.-C., Harvey, P., Evans, N. J., Mern, B., Koerner, D., Sargent, A., van Dishoeck, E. F., Allen, L., Blake, G., Brooke, T., Chapman, N., Huard, T., Lai, S.-P., Mundy, L., Myers, P. C., Spiesman, W., \& Wahhaj, Z. 2007, The Astrophysical Journal, 667, 308

Clayton, R. N., Mayeda, T. K., Gooding, J. L., Keil, K., \& Olsen, E. J. 1981, in Lunar and Planetary Institute Science Conference Abstracts, Vol. 12, 154156

Connolly, H. C., Hewins, R. H., Ash, R. D., Zanda, B., Lofgren, G. E., \& Bourot-Denise, M. 1994, , Published online: 08 September 1994; | doi:10.1038/371136a0, 371, 136

Desch, S. J., \& Cuzzi, J. N. 2000, Icarus, 143, 87

Fleming, T. P., Stone, J. M., \& Hawley, J. F. 2000, The Astrophysical Journal, 530, 464

Fujii, Y. I., Okuzumi, S., \& Inutsuka, S. 2011, The Astrophysical Journal, 743, 53

Gammie, C. F. 1996, The Astrophysical Journal, 457,355

Gardiner, T. A., \& Stone, J. M. 2005, Journal of Computational Physics, 205, 509

—. 2008, Journal of Computational Physics, 227, 4123

Grach, V., Semenov, V., \& Trakhtengerts, V. 2010, in 38th COSPAR Scientific Assembly, Vol. 38, 1521

Güttler, C., Blum, J., Zsom, A., Ormel, C. W., \& Dullemond, C. P. 2010, Astronomy and Astrophysics, 513, 56

Hawley, J. F., Gammie, C. F., \& Balbus, S. A. 1995, The Astrophysical Journal, 440, 742

Hayashi, C., Nakazawa, K., \& Nakagawa, Y. 1985, IN: Protostars and planets II (A86-12626 0390). Tucson, AZ, University of Arizona Press, 1985, p. 1100-1153., -1, 1100
Hernndez, J., Hartmann, L., Calvet, N., Jeffries, R. D., Gutermuth, R., Muzerolle, J., \& Stauffer, J. 2008, The Astrophysical Journal, 686, 1195

Horanyi, M., Morfill, G., Goertz, C. K., \& Levy, E. H. 1995, Icarus, 114, 174

Ilgner, M., \& Nelson, R. P. 2006, Astronomy and Astrophysics, 455, 731

Inutsuka, S., \& Sano, T. 2005, The Astrophysical Journal Letters, 628, L155

Jones, R. H., \& Danielson, L. R. 1997, Meteoritics \& Planetary Science, 32, 753760

Lofgren, G. 1989, Geochimica et Cosmochimica Acta, 53, 461470

Muranushi, T. 2010, Monthly Notices of the Royal Astronomical Society, 401, 2641

Okuzumi, S. 2009, The Astrophysical Journal, 698,1122

Ormel, C. W., \& Cuzzi, J. N. 2007, Astronomy and Astrophysics, 466, 413

Pilipp, W., Hartquist, T. W., \& Morfill, G. E. 1992, The Astrophysical Journal, 387, 364

Rubin, A. E. 2005, Geochimica et cosmochimica acta, 69, 49074918

Sano, T., Inutsuka, S., \& Miyama, S. M. 1998, The Astrophysical Journal Letters, 506, L57

Sano, T., Miyama, S. M., Umebayashi, T., \& Nakano, T. 2000, The Astrophysical Journal, 543,486

Shakura, N. I., \& Sunyaev, R. A. 1973, Astronomy and Astrophysics, 24, 337

Sodha, M. S., Misra, S., \& Mishra, S. K. 2009, Physics of Plasmas, 16, 123705

Suzuki, T. K., Muto, T., \& Inutsuka, S. 2010, The Astrophysical Journal, 718, 1289

Umebayashi, T., \& Nakano, T. 1980, Publications of the Astronomical Society of Japan, 32, 405

—. 2009, The Astrophysical Journal, 690, 69 
Wada, K., Tanaka, H., Suyama, T., Kimura, H., \& Yamamoto, T. 2008, The Astrophysical Journal, 677,1296

Wardle, M. 2007, Astrophysics and Space Science, 311,35

Wardle, M., \& Salmeron, R. 2012, Monthly Notices of the Royal Astronomical Society, 422, 2737

Weidenschilling, S. J. 1997, in Lunar and Planetary Institute Science Conference Abstracts, Vol. 28, 1515

Wettlaufer, J. S. 2010, The Astrophysical Journal, 719,540

This 2-column preprint was prepared with the AAS IATEX macros v5.2. 


\begin{tabular}{|c|c|c|c|c|c|c|c|c|}
\hline runID & $\mathrm{t}$ & $\beta$ & $\frac{J_{\text {crit }}}{J_{\text {eqp }}}$ & $R_{\mathrm{M}}$ & $\left\langle\frac{B^{2}}{8 \pi P_{0}}\right\rangle$ & $\left\langle\frac{-B_{x} B_{y}}{4 \pi P_{0}}\right\rangle$ & $\left\langle\frac{\rho v_{x} \delta v_{y}}{P_{0}}\right\rangle$ & $\left\langle\frac{J^{2}}{J_{\mathrm{eqp}}^{2}}\right\rangle^{0.5}$ \\
\hline 3160 & 0 & 400 & $\infty$ & $\infty$ & $(1.99 \pm 0.60) \times 10^{-1}$ & $(1.05 \pm 0.37) \times 10^{-1}$ & $(3.01 \pm 1.49) \times 10^{-2}$ & $(2.04 \pm 0.24) \times 10^{1}$ \\
\hline 10 & 0 & 800 & $\infty$ & $\infty$ & $(1.64 \pm 0.69) \times 10^{-1}$ & $(8.27 \pm 3.65) \times 10^{-2}$ & $(2.26 \pm 0.96) \times 10^{-2}$ & $(1.94 \pm 0.30) \times 10^{1}$ \\
\hline 220 & 0 & 1600 & $\infty$ & $\infty$ & $(1.94 \pm 0.61) \times 10^{-1}$ & $(9.47 \pm 3.19) \times 10^{-2}$ & $(2.34 \pm 0.84) \times 10^{-2}$ & $(2.07 \pm 0.26) \times 10^{1}$ \\
\hline 3180 & 0 & 3200 & $\infty$ & $\infty$ & $(1.30 \pm 0.27) \times 10^{-1}$ & $(6.08 \pm 1.47) \times 10^{-2}$ & $(1.68 \pm 0.52) \times 10^{-2}$ & $(1.85 \pm 0.18) \times 10^{1}$ \\
\hline 3612 & 0 & 6400 & $\infty$ & $\infty$ & $(7.16 \pm 2.57) \times 10^{-2}$ & $(3.14 \pm 0.85) \times 10^{-2}$ & $(8.28 \pm 2.51) \times 10^{-3}$ & $(1.51 \pm 0.15) \times 10^{1}$ \\
\hline 3616 & 0 & 12800 & $\infty$ & $\infty$ & $(3.61 \pm 0.83) \times 10^{-2}$ & $(1.70 \pm 0.35) \times 10^{-2}$ & $(6.15 \pm 2.40) \times 10^{-3}$ & $(1.24 \pm 0.10) \times 10^{1}$ \\
\hline 3620 & 0 & 25600 & $\infty$ & $\infty$ & $(3.61 \pm 0.84) \times 10^{-2}$ & $(1.63 \pm 0.36) \times 10^{-2}$ & $(5.40 \pm 1.84) \times 10^{-3}$ & $(1.22 \pm 0.09) \times 10^{1}$ \\
\hline 3292 & 0 & 400 & 1.0 & 0.6 & $(2.92 \pm 1.56) \times 10^{-1}$ & $(1.67 \pm 1.04) \times 10^{-1}$ & $(4.81 \pm 3.63) \times 10^{-2}$ & $(2.10 \pm 0.43) \times 10^{1}$ \\
\hline 3293 & $16 \pi / \Omega$ & 400 & 1.0 & 0.6 & $(2.74 \pm 1.43) \times 10^{-1}$ & $(1.62 \pm 0.99) \times 10^{-1}$ & $(4.63 \pm 3.05) \times 10^{-2}$ & $(2.06 \pm 0.48) \times 10^{1}$ \\
\hline 3294 & $18 \pi / \Omega$ & 400 & 1.0 & 0.6 & $(2.07 \pm 0.71) \times 10^{-1}$ & $(1.21 \pm 0.44) \times 10^{-1}$ & $(3.68 \pm 1.62) \times 10^{-2}$ & $(1.88 \pm 0.27) \times 10^{1}$ \\
\hline 3295 & $20 \pi / \Omega$ & 400 & 1.0 & 0.6 & $(2.34 \pm 0.82) \times 10^{-1}$ & $(1.36 \pm 0.54) \times 10^{-1}$ & $(3.86 \pm 1.80) \times 10^{-2}$ & $(2.00 \pm 0.30) \times 10^{1}$ \\
\hline
\end{tabular}

\begin{tabular}{|c|c|c|c|c|c|c|c|c|}
\hline 3352 & 0 & 400 & 1.0 & 0.2 & $(2.50 \pm 0.00) \times 10^{-3}$ & $(8.24 \pm 6.55) \times 10^{-13}$ & $(4.83 \pm 2.58) \times 10^{-7}$ & $(4.11 \pm 0.94) \times 10^{-3}$ \\
\hline 3353 & $16 \pi / \Omega$ & 400 & 1.0 & 0.2 & $(2.03 \pm 1.27) \times 10^{-1}$ & $(1.27 \pm 0.86) \times 10^{-1}$ & $(4.07 \pm 2.99) \times 10^{-2}$ & $(1.50 \pm 0.60) \times 10^{1}$ \\
\hline 3354 & $18 \pi / \Omega$ & 400 & 1.0 & 0.2 & $(2.21 \pm 0.98) \times 10^{-1}$ & $(1.33 \pm 0.50) \times 10^{-1}$ & $(4.68 \pm 2.68) \times 10^{-2}$ & $(1.66 \pm 0.41) \times 10^{1}$ \\
\hline 3355 & $20 \pi / \Omega$ & 400 & 1.0 & 0.2 & $(2.52 \pm 1.65) \times 10^{-1}$ & $(1.56 \pm 1.02) \times 10^{-1}$ & $(4.57 \pm 2.72) \times 10^{-2}$ & $(1.67 \pm 0.59) \times 10^{1}$ \\
\hline 3348 & 0 & 400 & 10.0 & 0.2 & $(2.50 \pm 0.00) \times 10^{-3}$ & $(8.24 \pm 6.55) \times 10^{-13}$ & $(4.83 \pm 2.58) \times 10^{-7}$ & $(4.11 \pm 0.94) \times 10^{-3}$ \\
\hline 3349 & $16 \pi / \Omega$ & 400 & 10.0 & 0.2 & $(2.50 \pm 0.00) \times 10^{-3}$ & $(1.77 \pm 1.01) \times 10^{-7}$ & $(9.90 \pm 7.50) \times 10^{-5}$ & $(3.60 \pm 0.54) \times 10^{-2}$ \\
\hline 3350 & $18 \pi / \Omega$ & 400 & 10.0 & 0.2 & $(2.50 \pm 0.00) \times 10^{-3}$ & $(2.31 \pm 1.64) \times 10^{-6}$ & $(6.06 \pm 9.16) \times 10^{-5}$ & $(3.49 \pm 0.54) \times 10^{-2}$ \\
\hline 3351 & $20 \pi / \Omega$ & 400 & 10.0 & 0.2 & $(2.50 \pm 0.00) \times 10^{-3}$ & $(4.85 \pm 3.58) \times 10^{-7}$ & $(6.62 \pm 8.64) \times 10^{-5}$ & $(3.43 \pm 0.36) \times 10^{-2}$ \\
\hline
\end{tabular}

Table 4: Statistics of the local simulations abridged. Each run is labeled by an integer. The re-start time is in the second column. Next three columns indicate the initial magnetic field strength, the critical current, and the magnetic Reynolds number. The physical quantity are represented in terms of the time average and standard deviation of the space average, i.e. $A$ is in the format $\overline{\langle A\rangle} \pm\left({\overline{\langle A\rangle^{2}}}^{2} \overline{\langle A\rangle}^{2}\right)^{0.5}$

. In this table are runs for ideal MHD, runs in Figure 2 that represent the behavior in (a) active, (b) sustained, and (c) dead zones, runs that constitute sustained-dead zone boundaries for $\beta=400,3200$ 


\begin{tabular}{|c|c|c|c|c|c|c|c|c|}
\hline runID & $\mathrm{t}$ & $\beta$ & $\frac{J_{\text {crit }}}{J_{\text {eqp }}}$ & $R_{\mathrm{M}}$ & $\left\langle\frac{B^{2}}{8 \pi P_{0}}\right\rangle$ & $\left\langle\frac{-B_{x} B_{y}}{4 \pi P_{0}}\right\rangle$ & $\left\langle\frac{\rho v_{x} \delta v_{y}}{P_{0}}\right\rangle$ & $\left\langle\frac{J^{2}}{J_{\mathrm{eqp}}^{2}}\right\rangle^{0.5}$ \\
\hline 3352 & 0 & 400 & 1.0 & 0.2 & $(2.50 \pm 0.00) \times 10^{-3}$ & $(8.24 \pm 6.55) \times 10^{-13}$ & $(4.83 \pm 2.58) \times 10^{-7}$ & $(4.11 \pm 0.94) \times 10^{-3}$ \\
\hline 3353 & $16 \pi / \Omega$ & 400 & 1.0 & 0.2 & $(2.03 \pm 1.27) \times 10^{-1}$ & $(1.27 \pm 0.86) \times 10^{-1}$ & $(4.07 \pm 2.99) \times 10^{-2}$ & $(1.50 \pm 0.60) \times 10^{1}$ \\
\hline 3452 & 0 & 400 & 0.9 & 0.06 & $(2.50 \pm 0.00) \times 10^{-3}$ & $(3.05 \pm 2.55) \times 10^{-13}$ & $(4.29 \pm 2.47) \times 10^{-7}$ & $(2.22 \pm 0.52) \times 10^{-3}$ \\
\hline 3453 & $16 \pi / \Omega$ & 400 & 0.9 & 0.06 & $(2.50 \pm 0.00) \times 10^{-3}$ & $(1.22 \pm 0.75) \times 10^{-8}$ & $(2.29 \pm 1.58) \times 10^{-4}$ & $(3.02 \pm 0.46) \times 10^{-2}$ \\
\hline 3448 & 0 & 400 & 0.3 & 0.06 & $(2.50 \pm 0.00) \times 10^{-3}$ & $(3.05 \pm 2.55) \times 10^{-13}$ & $(4.29 \pm 2.47) \times 10^{-7}$ & $(2.22 \pm 0.52) \times 10^{-3}$ \\
\hline 3449 & $16 \pi / \Omega$ & 400 & 0.3 & 0.06 & $(2.29 \pm 2.53) \times 10^{-1}$ & $(1.43 \pm 1.71) \times 10^{-1}$ & $(4.61 \pm 4.95) \times 10^{-2}$ & $(1.39 \pm 0.90) \times 10^{1}$ \\
\hline 3436 & 0 & 400 & 0.3 & 0.02 & $(2.50 \pm 0.00) \times 10^{-3}$ & $(9.13 \pm 12.07) \times 10^{-13}$ & $(1.20 \pm 1.54) \times 10^{-7}$ & $(3.43 \pm 2.16) \times 10^{-4}$ \\
\hline 3437 & $16 \pi / \Omega$ & 400 & 0.3 & 0.02 & $(2.50 \pm 0.00) \times 10^{-3}$ & $(2.39 \pm 1.08) \times 10^{-9}$ & $(8.02 \pm 6.85) \times 10^{-5}$ & $(1.17 \pm 0.23) \times 10^{-2}$ \\
\hline 3432 & 0 & 400 & 0.1 & 0.02 & $(2.50 \pm 0.00) \times 10^{-3}$ & $(9.55 \pm 12.38) \times 10^{-13}$ & $(8.32 \pm 8.82) \times 10^{-8}$ & $(2.91 \pm 1.54) \times 10^{-4}$ \\
\hline 3433 & $16 \pi / \Omega$ & 400 & 0.1 & 0.02 & $(2.00 \pm 2.42) \times 10^{-1}$ & $(1.14 \pm 1.50) \times 10^{-1}$ & $(3.54 \pm 4.61) \times 10^{-2}$ & $(1.23 \pm 0.98) \times 10^{1}$ \\
\hline
\end{tabular}

\begin{tabular}{|c|c|c|c|c|c|c|c|c|}
\hline 3372 & 0 & 3200 & 1.0 & 0.2 & $(1.11 \pm 0.62) \times 10^{-1}$ & $(5.22 \pm 2.79) \times 10^{-2}$ & $(1.39 \pm 0.68) \times 10^{-2}$ & $(1.58 \pm 0.31) \times 10^{1}$ \\
\hline 3373 & $16 \pi / \Omega$ & 3200 & 1.0 & 0.2 & $(1.00 \pm 0.41) \times 10^{-1}$ & $(4.29 \pm 1.61) \times 10^{-2}$ & $(9.90 \pm 3.04) \times 10^{-3}$ & $(1.52 \pm 0.24) \times 10^{1}$ \\
\hline 3460 & 0 & 3200 & 0.9 & 0.06 & $(3.12 \pm 0.00) \times 10^{-4}$ & $(3.02 \pm 1.86) \times 10^{-12}$ & $(4.68 \pm 2.21) \times 10^{-7}$ & $(1.89 \pm 0.39) \times 10^{-3}$ \\
\hline 3461 & $16 \pi / \Omega$ & 3200 & 0.9 & 0.06 & $(3.35 \pm 0.24) \times 10^{-4}$ & $(9.82 \pm 10.64) \times 10^{-6}$ & $(3.36 \pm 3.24) \times 10^{-5}$ & $(5.07 \pm 2.78) \times 10^{-2}$ \\
\hline 3456 & 0 & 3200 & 0.3 & 0.06 & $(3.12 \pm 0.00) \times 10^{-4}$ & $(3.02 \pm 1.86) \times 10^{-12}$ & $(4.68 \pm 2.21) \times 10^{-7}$ & $(1.89 \pm 0.39) \times 10^{-3}$ \\
\hline 3457 & $16 \pi / \Omega$ & 3200 & 0.3 & 0.06 & $(1.19 \pm 0.48) \times 10^{-1}$ & $(5.44 \pm 1.88) \times 10^{-2}$ & $(1.42 \pm 0.51) \times 10^{-2}$ & $(1.65 \pm 0.26) \times 10^{1}$ \\
\hline 3444 & 0 & 3200 & 0.3 & 0.02 & $(3.12 \pm 0.00) \times 10^{-4}$ & $(8.60 \pm 6.12) \times 10^{-13}$ & $(5.18 \pm 2.54) \times 10^{-7}$ & $(1.55 \pm 0.35) \times 10^{-3}$ \\
\hline 3445 & $16 \pi / \Omega$ & 3200 & 0.3 & 0.02 & $(9.38 \pm 4.06) \times 10^{-2}$ & $(4.50 \pm 1.88) \times 10^{-2}$ & $(1.15 \pm 0.46) \times 10^{-2}$ & $(1.29 \pm 0.27) \times 10^{1}$ \\
\hline 3440 & 0 & 3200 & 0.1 & 0.02 & $(3.12 \pm 0.00) \times 10^{-4}$ & $(8.60 \pm 6.12) \times 10^{-13}$ & $(5.18 \pm 2.54) \times 10^{-7}$ & $(1.55 \pm 0.35) \times 10^{-3}$ \\
\hline 3441 & $16 \pi / \Omega$ & 3200 & 0.1 & 0.02 & $(8.15 \pm 3.91) \times 10^{-2}$ & $(3.79 \pm 1.70) \times 10^{-2}$ & $(1.05 \pm 0.50) \times 10^{-2}$ & $(1.41 \pm 0.23) \times 10^{1}$ \\
\hline
\end{tabular}

Table 4: (continued) 